\title{
Two factors but one dimension: An alternative view at the structure of mood and emotion
}

\author{
Yvonnick Noel, Florence Fevrier \& Anne Deflandre \\ University of Brittany, Rennes 2 (France) \\ Department of Psychology
}

\begin{abstract}
This paper argues that well-established components of human affect (i.e. positive vs. negative valence and high vs. low arousal) are dynamically and non-linearly related. This main hypothesis is based on the two more specific hypotheses that i) when subject to environmental stressors, an organism in an initially stable positive mood state will increase its arousal level, to manage the organism-environment relationship (i.e. a state of alertness, Lazarus and Folkman 1984), and ii) arousal is expected to decrease when the intensity of negative feedback bypasses some reasonable level, as is well known from depression and learned helplessness studies (Seligman, 1972). Put together, both assumptions lead to a model of affect where arousal varies as a single-peaked function of valence, conceived of as a bipolar (low arousal positive vs. low arousal negative) dimension. It is argued that traditional factor techniques are not appropriate to analyze such nonlinear relationships, and an unfolding item response model is proposed that models both emotional between-subject heterogeneity and within-subject variability. This model shows a good fit on self-rating data, in an emotional change study (Study $4, N=746$ ), but also shed a new light on some puzzling issues in the dimensional modeling of emotion: Why positive and negative emotions may have appeared as orthogonal factors (Study 1 and 2), why arousal and valence may have shown a V-shape pattern of relationship (study 3), and why factor analyses of emotional items tend to yield circumplex patterns of loadings. A new and easy-to-compute practical measure of mood, derived from the model, is also proposed.
\end{abstract}

Keywords: Emotion, mood, valence, arousal, factor analysis, unfolding analysis.

\section{Introduction}

The search for a structure of affect has retained much attention in psychological and biological research over the last decades. But despite a large amount of research, and some psychometric sophistication, current models of affect leave a number of questions unanswered, or at least provide contradictory responses, in particular as far as dimensionality and polarity of emotion are concerned. Tracing back the historical roots of modern ideas on affect and emotion may help better understand their conceptual limitations, and suggest new ways of thinking.

A classical approach to the structure of emotion has been to distinguish between discrete categories of emotions. Plutchik (2002, p. 69) reports that it may be found in ancient Hindu philosophical texts (eight basic emotions were

Correspondence concerning this article should be sent to: Yvonnick Noel, PhD., University of Brittany Rennes 2, Department of Psychology, Place du Recteur Henri Le Moal, CS 24307, F-35043 Rennes Cedex, France. E-mail: yvonnick.noel@uhb.fr. Portions of this paper have been presented at the 21st International Meeting of Differential Psychology, June 2014, Paris, France. distinguished: love, laughter, sorrow, anger, fear, perseverance, disgust, amazement). In the Middle Ages, the French philosopher Descartes (1649) considered everyday "passions" as mixtures of six basic emotions (love, hatred, desire, joy, sadness, and admiration) when Spinoza (1677), in the third part of his Ethics, presents affects as springing from only three basic emotions (joy, sorrow, desire). The English philosopher Hobbes distinguished between seven elementary passions (appetite, desire, love, aversion, hate, joy and grief). Darwin (1872) identified seven clusters of emotions both in human and animals. Early in the XXth century, the British psychologist William McDougall (1921) regarded complex emotions as mixtures of seven primary emotions (fear, disgust, wonder, anger, subjection, elation, and tender feelings).

As of the 60s, an intense line of research, beginning with Tomkins (1962), who assumed eight basic emotions, provided evidence for cross-cultural, stable, basic emotions. In the line of Tomkins' works, but based on a search for well differentiated facial expressions and specific autonomic substrates, a set of seven (happiness, surprise, sadness, disgust, angriness, fear, contempt), then six basic categories (joy, surprise, sadness, disgust, angriness, fear) was established (Ekman \& Friesen, 1971; Izard, 1971).

In categorical models of emotion, the common experience 
of mixed feelings is thought of as a mixture of the basic emotions. But besides the fact that the number of basic emotions to be retained may in itself be somewhat controversial, the categorical approach may fail to account for systematic correlations observed between some of them. A more recent approach has been to try to model affect as determined by a set of latent factors. The emergence of factor models of affect may be viewed as an attempt to better capture that different emotions may be experienced with continuously (co)varying intensities. In an early attempt, Cattell (1957) reported ten factors of expressions of emotion, and he defined them as basic emotions with their own adaptive goals. Conceptually, this may be viewed as a transitional step, as in this presentation, factors are still theoretically manipulated as separate, although potentially combined, entities, very much like categories.

But the factor approach also offers a description of how different emotional feelings may be experienced at the same time (orthogonal factors) or not (bipolar factors). A strong research trend emerged in the 80 s that proposed to subsume the consistent positive correlations between positive affective states on the one hand (e.g. "happy", "delighted"), and negative affective states on the other hand (e.g. "sad", "depressed"), under two dominant higher order and uncorrelated factors: Negative Affect and Positive Affect (Tellegen, Watson, \& Clark, 1999; Watson \& Tellegen, 1985, 1999). These two higher order factors have been reported to emerge consistently across diverse sets of descriptors, time frames, response formats, and languages (Watson \& Clark, 1997; Watson \& Tellegen, 1999). But the consequences of this line of thinking are puzzling, as considering positive and negative affect as two independent unipolar factors implies that a person may simultaneously feel in a high positive and high negative mood state. In a well-known controversy, it was shown (Russell, 1979; Russell \& Carroll, 1999) that when in addition to positive and negative mood items, a set of arousal descriptors (e.g. "alert", "stimulated") was taken into account, positive and negative affects appeared as a single bipolar dimension (named emotional valence) in factor analyses, arousal items forming a second orthogonal factor. This nontrivial variation in outcome, in itself, could have led to think that both aspects of affect, now commonly named valence and arousal, might actually not be independent (in a sense to be clarified in the next sections).

This debate may be viewed as an attempt, albeit in the limited framework of linear models, to clarify the relationships between constructs that repeatedly emerged from factor analytic studies (positive affect, negative affect, high activation, low activation). In this framework, however, the issue of between-factor relationships can only be addressed in terms of linear dimensionality and polarity, leading to questions such as: Should one, two or more linear components be retained? Do positive and negative affect form two independent (i.e. uncorrelated) components or a single bipolar dimension (i.e. there is a perfect negative correlation between them)? Do arousal and valence form a set of orthogonal factors?

Although a consensus seems to have emerged during the 90s in favor of a two-factor model (valence and arousal), for which differentiated neuro-physiological substrates have been proposed (Posner et al., 2009), clues exist that valence and arousal might actually interact, at least at a functional level, in their impact on a number of cognitive processes. Such an interaction has been found in the ongoing emotional processing of words in recognition and reading tasks, where faster reaction times are systematically found for negativeactive and positive-passive words (Citron, Gray, Critchley, Weekes, \& Ferstl, 2014; Citron, Weekes, \& Ferstl, 2014; Robinson, Storbeck, Meier, \& Kirkeby, 2004), in the access to awareness during perception tasks (Sheth \& Pham, 2008), in emotion-enhanced memory where the effect of arousal appears to be moderated by the valence of stimuli (Kang, Wang, Surina, \& Lu, 2014), to name but a few.

At a more structural level, recent works have also brought empirical evidence of a nonlinear relationship between arousal and valence. Kuppens, Tuerlinckx, Russell, and Barrett (2013) systematically compared constant, linear, Vshaped and inverted-U shaped models of relationship between them. They provided empirical evidence in favor of a V-shaped model, in concordance with what had been previously reported by Lang, Bradley and colleagues, based on averaged ratings of Pleasure and Arousal evoked by affectively laden images from the International Affective Picture System (IAPS) (Bradley \& Lang, 2007; Lang, Bradley, \& Cuthbert, 2008).

The present paper tackles the same problem from a different perspective. The main hypothesis to be examined is that valence and arousal are dynamically and non-linearly related. This main hypothesis is based on two more specific hypotheses. In the line of Lazarus and Folkman (1984)'s Transactional Model of Stress, we first hypothesize that increasing arousal is biologically adaptive when negative feedbacks are received from the environment, because something need to be changed in the organism-environment relationship (i.e. a state of alertness). So empirically an increase in arousal should be observed when affect evolves from a stable positive equilibrium towards a negative valence. But on the other hand, arousal is expected to decrease when the intensity of negative feedback bypasses a reasonable level, as is well known from learned helplessness studies (Seligman, 1972). Put together, both assumptions leads to a model of affect where arousal varies as a single-peaked function of valence, conceived of as a bipolar (low arousal positive vs. low arousal negative) dimension. Beyond categories and factors, conceiving of valence and activation as interacting processes within a common dynamical system with a single control variable will provide another view at the structure of 
affect.

As many questions on the structure of mood actually arise in very similar terms in the study of emotions, both will be subsumed under the term "affect" in this paper, although both are usually conceptually distinguished (Beedie, Terry, Lane, \& Devonport, 2011). Emotion has been defined as a more structured, in-situation, psycho-physiological response to an event, experienced with a potentially high intensity, in relation to the needs or survival of the organism (Watson, 2000, p. 3). By contrast, moods are conceived of as transient episodes of feeling and affect, experienced both on a longer time scale and on lower intensity levels. This insightful distinction will be given some further support in the present paper, and will be formulated in more functional and dynamical terms, within the model to be proposed.

The first part of this paper is dedicated to a full reanalysis of the structure of positive and negative valence. In particular, from an in-depth psychometric discussion about the use of factor analysis and principal component analysis in affect studies, we provide new arguments as to why negative vs. positive affect valence should be modeled as a bipolar construct, in the line of Russell (1979), but also to an explanation of why they may have appeared as two orthogonal factors in a number of previous studies. In a second part, we elaborate on a new model of interaction between valence and arousal, and reanalyze a number of classical results in the light of this new hypothesis. In particular, an explanation of why affect items appear as circumplex patterns in factor loading plots, and why arousal and valence ratings of IAPS images display a boomerang-shaped pattern is provided. Finally, a test-retest study of emotional change in a stressing situation is presented, that directly tests the single-peaked relationship hypothesis. The model has strong consequences both at the conceptual and practical levels, in particular as far as emotion assessment is concerned, and these are detailed in the discussion.

\section{Bipolarity of the valence dimension}

Before looking at the valence-arousal relationship, we deal in this section with the simpler issue of a dimensional representation of positive and negative affect, and some related methodological problems, temporarily setting aside arousal items. This is an important first step to both understand why so different and somewhat contradictory representations have been found, and to understand the relationship to arousal, in a second step.

\section{Factors are not dimensions}

Most studies on the factor structure of emotion have used factor analysis (FA) or principal component analysis (PCA). Although strictly speaking PCA is not a statistical model, both techniques have yielded very similar results and both suffer from the limitations to be discussed in this section, albeit for different reasons. These techniques actually rely on two fundamental assumptions: i) data are numeric, ii) variables are linearly related. Although seemingly trivial, the consequences of these assumptions have been somewhat overlooked.

First, factor techniques are very commonly used with Likert data in psychology and, notwithstanding the fact that Likert data are not numeric but ordinal by nature, they should be regarded as bounded data. Application of linear models to bounded data may is known to potentially yield misleading results (Smithson \& Verkuilen, 2006; Verkuilen \& Smithson, 2012), and this boundary effect has already been pointed out as a potential source of problematic floor effects in the measurement of emotion. For example, an item such as "fearful" usually exhibits a very low mean, and because of the bounded nature of collected responses, this also implies a very low variance (Ekkekakis, Hall, \& Petruzzello, 2005; Ekkekakis, Hall, Petruzzello, et al., 1999; Jerome et al., 2002). This mean-variance relationship is theoretically expected, given the bounded nature of responses, and very badly modeled by homoscedastic linear models. In this context, the usual practice of standardizing response variables is likely to further complicate the picture, as variance does contain meaningful information. A dual model, on both expectation and variance (and their relationship), should be made explicit, very much like a binomial logistic regression model incorporates specific constraints on the expectation function (taking into account the bounded nature of binary data), on the variance function (taking into account that variance is low for extreme values of the linear term) and the (quadratic in this case) relationship between both.

Second, in a linear vector space, variables relationships are represented and measured as angles, and independence is synonymous with orthogonality. But linear independence should by no means be confounded with statistical independence, and so neither with psychological independence nor discriminant validity. If two psychological phenomena are nonlinearly related, their statistical dependence will not be correctly uncovered by linear factor techniques (e.g. a perfect quadratic relationship would translate into a null correlation).

One way to clarify things in the analysis of these problems is to adopt a generative approach, i.e. to specify an explicit response function, as is common in Item Response Theory (Embretson \& Reise, 2000; van der Linden \& Hambleton, 1997). From a model of how responses are related to a common latent dimension, for all items in a questionnaire, a model of the relationship between any two response variables (or their conditional expectations at some trait level) can be deduced. As a first example, let us consider the linear congeneric response model (Ferrando, 2001; Mellenbergh, 1994) for two hypothetical items in a mood questionnaire, 
1a. Linear response functions

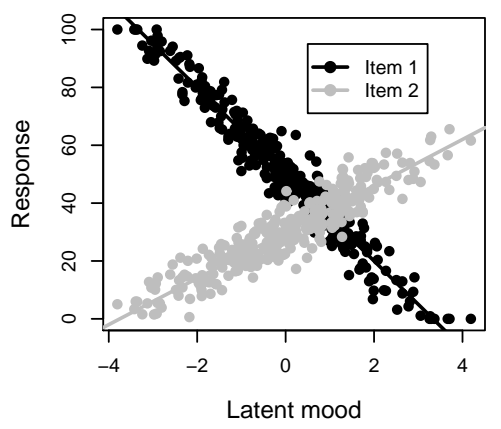

2a. Logistic response functions (I)

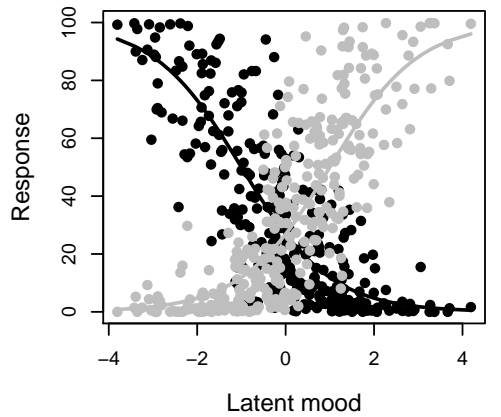

3a. Logistic response functions (II)

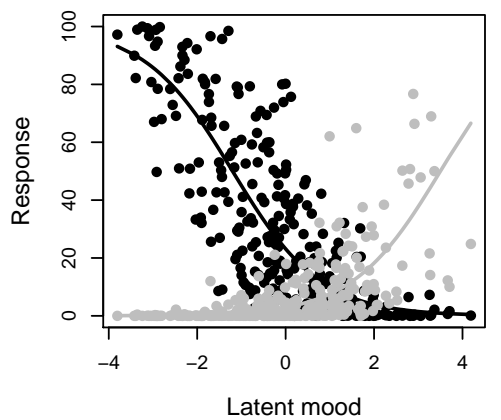

1b. Correlogram

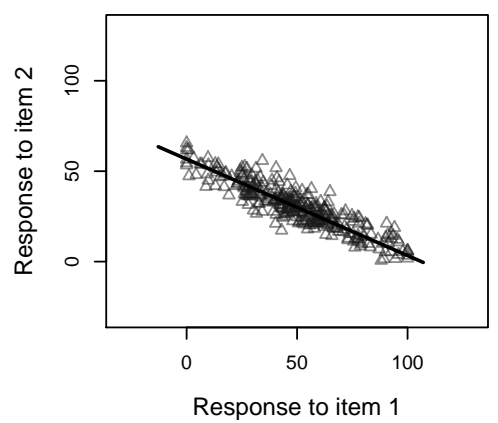

2b. Correlogram

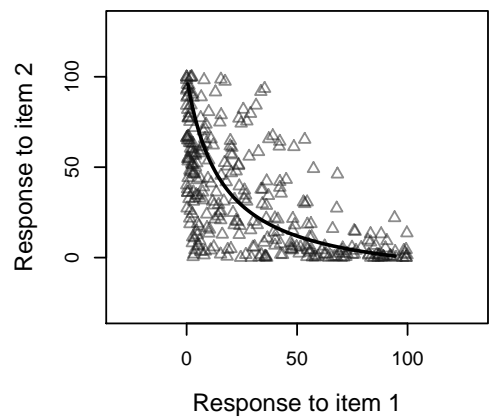

3b. Correlogram

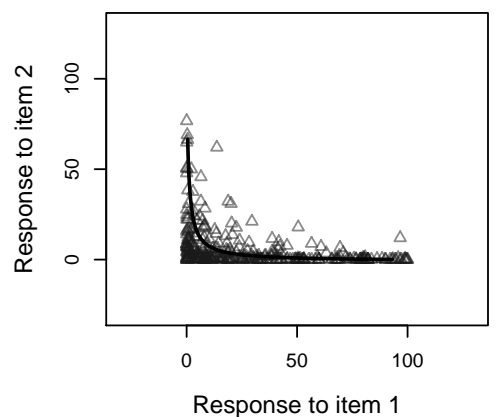

1c. PCA: Linear case

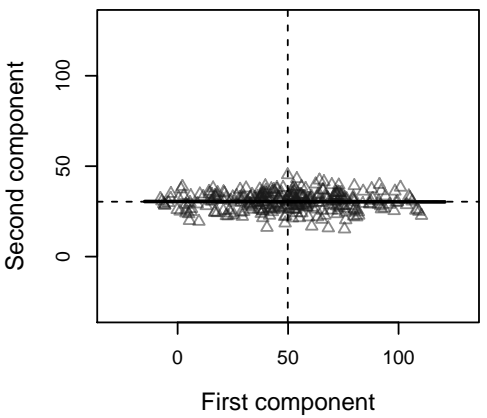

2c. PCA: Logistic case (I)

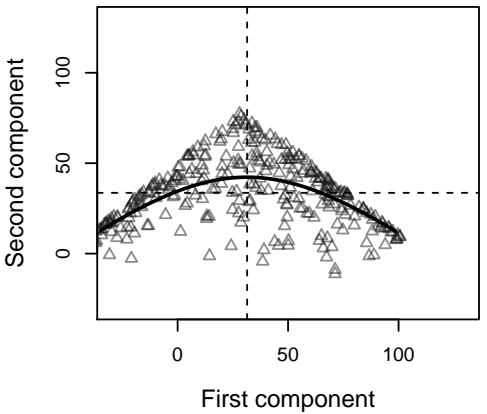

3c. PCA: Logistic case (II)

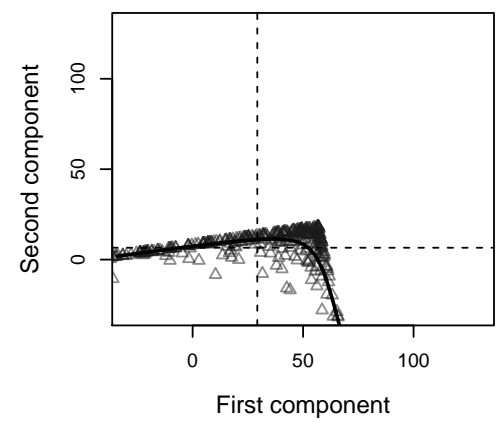

Figure 1. The linear response model (upper row) and a logistic response model for two sample items, with close (middle row, $\delta_{1}=-1, \delta_{2}=1$ ) and more distant locations (bottom row, $\delta_{1}=-1.2, \delta_{2}=3.5$ ). In each case, the response functions, the resulting between-variable relationships and the unscaled PCA solution are shown on left, middle and right panels, respectively.

and the corresponding response variables $X_{j}(j=1,2)$ :

$$
X_{i j}=\mu_{j}+\lambda_{j} \theta_{i}+\epsilon_{i j}
$$

where $\mu_{j}$ and $\lambda_{j}$ are item mean and factor loading (or discrimination) respectively. In this model, the measurement error $\epsilon_{i j}$ is assumed to have zero expectation, and the conditional expected response is linearly related to the latent attitude (or mood in this context):

$$
E\left(X_{i j} \mid \theta_{i}\right)=\mu_{j}+\lambda_{j} \theta_{i}
$$

Denoting by $\eta_{i j}$ the expected response of subject $i$ to item $j(j=1,2)$, in the simple case of two items, a model of relationship between both expected responses is easily deduced from (2) as:

$$
\eta_{i 1}=\left(\frac{\lambda_{1}}{\lambda_{2}}\right) \eta_{i 2}+\left(\mu_{1}-\frac{\lambda_{1}}{\lambda_{2}} \mu_{2}\right) .
$$

The resulting dependence model is thus linear. Both representations (response functions and correlogram) for the linear response model are plotted on Figure 1 (upper row, panels 1a and 1b) for two simulated items where responses arbitrary range from 0 to $100\left(\mu_{1}=50, \lambda_{1}=-15, \mu_{2}=30, \lambda_{2}=8\right)$, 
with responses drawn from a $N\left(\eta_{i j}, 5^{2}\right)$ distribution. These items are constructed to reflect a bipolar construct, such that responses on either one are negatively related to responses on the other, as could be the case for opposite mood adjectives on a valence dimension (for instance "anxious" and "serene"). The corresponding PCA solution is plotted on panel 1c (to help compare with the original data, and without loss of generality, the PCA solution has been voluntarily left unscaled and uncentered). Because PCA looks for linear combinations of observed variables, PCA scores on the first factor are by construction linearly related to expected responses on all items simultaneously, which in turn are linearly related to true $\theta_{i}$ scores (Equation 2). In this case, factor techniques are legitimate and PCA will do a reasonable job at estimating latent scores through orthogonal projection onto the first principal component of the data cloud.

But a linear response model is actually not well-suited to the modeling of bounded responses: For some values of $\theta_{i}$ and $\lambda_{j}$, the model may well predict responses outside the response scale range, which is meaningless. Note that responses considered in this example are continuous, as would be the case if subjects had reported their mood on visual analogue scales. But the very same remarks would apply if Likert responses, considered as numeric, had been used (a common, albeit disputable, practice). An alternative is to consider a logistic expected response model, and a proper, bounded-support, distributional model, which explicitly takes response scale boundaries into account. For person $i$ and item $j$, the expected response (for data rescaled on the $[0 ; 1]$ interval) reads:

$$
\eta_{i j}=\frac{\exp \left(\theta_{i}-\delta_{j}\right)}{1+\exp \left(\theta_{i}-\delta_{j}\right)}
$$

where $\delta_{j}$ is an item location parameter on the latent dimension (defined as that attitude value for which a $50 \%$ agreement response is expected). Such a model, quite analogue to the Rasch model of measurement for binary data (Rasch, 1960), but here in the context of continuous responses, has been derived from a psychologically justified mechanism by Noel and Dauvier (2007), under the name Beta Response Model, and extended in Noel (2014). In the case of two items, the relationship between both expected responses is such that:

$$
\operatorname{logit}\left[\eta_{i 1}\right]=\operatorname{logit}\left[\eta_{i 2}\right]+\left(\delta_{2}-\delta_{1}\right)
$$

where logit[.] is the inverse logistic function. The resulting dependence model is no more linear, but logit-linear. Two logistic response functions, following Noel \& Dauvier's Beta Response Model, are shown on Figure 1 for two sample items with close locations (panel 2a, $\delta_{1}=-1$ and $\delta_{2}=1$ ) and more distant locations (panel 3a, $\delta_{1}=-1.2$ and $\delta_{2}=3.5$ ). The resulting correlograms are shown on panels $2 \mathrm{~b}$ and $3 \mathrm{~b}$ of
Figure 1, along with the bivariate expected curve. From the properties of PCA, finding principal components (i.e. linear combinations with normed coefficients) is nothing else than rotating these correlograms so that points are maximally dispersed on the horizontal axis. Clearly, no such rotation will ever linearize the relationship, as is apparent on panel 2c.

Although factor scores on the first component may give an acceptable approximation to latent scores (logistic case I), it should be clear enough that the second component is psychologically meaningless: PCA scores on the second component are non-linearly (and non monotonically) related to expected responses, which in turn are not linearly (through a logistic function here) related to true latent scores. As is apparent on the figure, variance on this second component is artificially inflated by this nonlinearity, and application of traditional factor techniques is clearly inappropriate. If ignored, this phenomenon may lead to retain a spurious additional factor to account for this extra dispersion.

In the third case (bottom row of Figure 1), item 2 is an extreme item and has low mean agreement. The floor effect dramatically increases, and this falsely orthogonalizes responses on both items (panel 3c), such that both response variables may erroneously appear as representing two orthogonal factors. The widespread use of varimax rotations is likely to make things still worse, as this procedure explicitly seeks such an item-factor specific affinity. So, depending on the inter-item distance $\delta_{1}-\delta_{2}$ on the latent dimension, both ends of what is intrinsically a bipolar construct may either be partially captured as a single bipolar factor or as two orthogonal factors by factor techniques.

This extra-factor phenomenon has been known for long in the psychometric literature, and already related to some specific nonlinear response process (Davison, 1977; Maraun \& Rossi, 2001; van Schuur \& Kiers, 1994). Still, it is largely ignored and not taken into account in many PCA and FA studies. As a consequence, artifactual factors are sometimes erroneously given substantial interpretations in psychological research.

After the raging debate between the two-factor and the bipolar model of emotional valence in the 80 s, one might have thought that some kind of consensus had been reached (Barrett \& Russell, 1998) on the fact that both solutions are actually equivalent, up to a $45^{\circ}$ rotation in the loading space (Feldman Barrett \& Russell, 1998). Some researchers may even have claimed that it is a matter of arbitrary choice (Yik, Russell, \& Barrett, 1999). But there have been a number of discordant voices over the years, to claim for example that "Happy" and "Sad" are not truly opposite (Rafaeli \& Revelle, 2006).

In a considerable effort to provide a non-ambiguous definition of bipolarity, Russell and Carroll (1999) discussed with much detail the question of which correlation value would reveal bipolarity. Their 2D and 3D correlogram plots 
(Figure 5, p. 16) on what happen between a "Sad" and "Happy" items when response formats are varied are very insightful and informative. But surprisingly, this does not lead them to question the very use of the correlation coefficient, when floor and ceiling effects are massive in the data they comment.

In the view proposed in this section, a scale will be said bipolar when the expected responses from two subsets of items are inverted monotonic (possibly nonlinear and shifted) functions of a common latent variable. Because monotonic does not necessarily mean linear, this definition encompasses those cases where truly opposite sets of variables display weak to moderate correlations.

This leads to an important distinction: Factors are not dimensions. When applied to truly unidimensional data, as was the case in the examples discussed above, and to satisfy common criteria of percentage of variance explained, factor techniques will likely extract too many factors as soon as a linear approximation to the response function is not acceptable. As is apparent on Figure 1 (panels 2c and 3c) correlograms will display curvilinear patterns as soon as the response functions are not strictly linear. Proper analysis of multivariate responses in these cases will require that a principal curve (not component) be extracted (Hastie \& Stuetzle, 1989; Tibshirani, 1992), to uncover the latent response function. This will be illustrated in the next section.

\section{Study 1: Fans, horseshoes and circumplexes}

To further illustrate the extra-factor phenomenon, and understand the meaning of principal components in these cases, we show in this section how truly unidimensional data may falsely appear as multifactorial in a more realistic situation where responses on more than 2 items are analyzed. Additionally, we want to make it clear that circumplex patterns are, at least partly, artefactual.

Simulated data. Responses from 300 simulated subjects have been generated following Noel \& Dauvier (2007)'s Beta Response Model (BRM). Subject's attitudes are drawn form a $N\left(0,1.5^{2}\right)$, and 8 items' locations are generated evenly spaced between -2.5 and +2.5 . For each item a precision (or inverse variance) parameter is drawn uniformly from $[0 ; 3]$ that controls the response dispersion around the logistic item characteristic curve (see Noel \& Dauvier, 2007 for details). The resulting data and item characteristic curves are plotted on Figure 2. The generated responses are drawn from a Beta distribution, so bounded by nature in $[0 ; 1]$, and the logistic response functions at the core of the BRM naturally take that particularity into account. The data have a clear unidimensional bipolar structure, the first four items having a decreasing response function, the last four having an increasing response function.

Analysis. The result of a standard scaled PCA on these simulated data is plotted on Figure 3 (left panel) and the Vari- max rotated solution is plotted on the right panel of the same figure. As a non-negligible amount of variance is borne by the second factor, and especially after a Varimax rotation, we could be led to distinguish and try to interpret two orthogonal factors, when a single true latent dimension exists. These plots display a fan-like or semi-circular pattern of loadings for both positive and negative subsets, which suggests that a subtle nonlinear dependency exists between loadings on both axes. Such patterns resemble patterns known as (semi) "circumplex" patterns, after Guttman (1954), that have repeatedly been observed in PCA studies of bipolar constructs (see Plutchik \& Conte, 1997, for a review), but also in correspondence analysis (Greenacre, 2007, p. 127) and multidimensional scaling studies (Everitt, 2005). This deserves an explanation, because it has a different meaning than what was discussed in the previous section for the curved patterns in the observation space.

Interpretation. To fully understand the origin of such loading patterns (and also why they have probably been a little bit overinterpreted), we first recall two well-known properties of PCA. First, on standardized data, factor loading $\lambda_{j k}$ of observed variable $\mathbf{x}_{j}(j=1, \ldots, p)$ on component $k$ $(k=1, \ldots, p)$ is the correlation of $\mathbf{x}_{j}$ with the $k$ th component. Second, very much like what holds in a multiple regression context, the square loading is the percentage of item variance explained by the component. So, because the set of all principal component form a full rank orthonormal basis in the variable space, we have that:

$$
\sum_{j=1}^{p} \lambda_{j k}^{2}=1
$$

The set of all variable loadings thus lie on the hypersphere of radius 1 . As a particular case, if a two-factor solution provided a perfect fit to the observed correlation matrix, then the pairs of factor loadings $\left(\lambda_{j 1}, \lambda_{j 2}\right)$ for all variables on the two first factors would lie on a unit-radius circle (i.e. $\lambda_{j 1}^{2}+\lambda_{j 2}^{2}=1$ ). In practice, if a two-factor solution provides an acceptable fit, but measurement error is present, then we may observe that $\lambda_{j 1}^{2}+\lambda_{j 2}^{2} \approx c$, with $0<c<1$. So, circular patterns are expected by construction in this context and have no psychological interpretation.

But what characterizes a circumplex from a more substantial point of view is that the curvilinear pattern of variables points reveals some kind of smooth gradation along a psychological dimension (e.g. from positive to negative valence), very much like a color wheel (Acton \& Revelle, 2004). From Equation 6, this indicates that moving along the true latent dimension, from positive to negative ends, the importance of the first factor in the explanation of observed responses will negatively covary with the importance of the second one, under the circular constraint (6). Formally, the nature of what "increases" or "decreases" in the reconstruction of $\mathbf{x}_{j}$ is borne by the set of principal components (ar- 

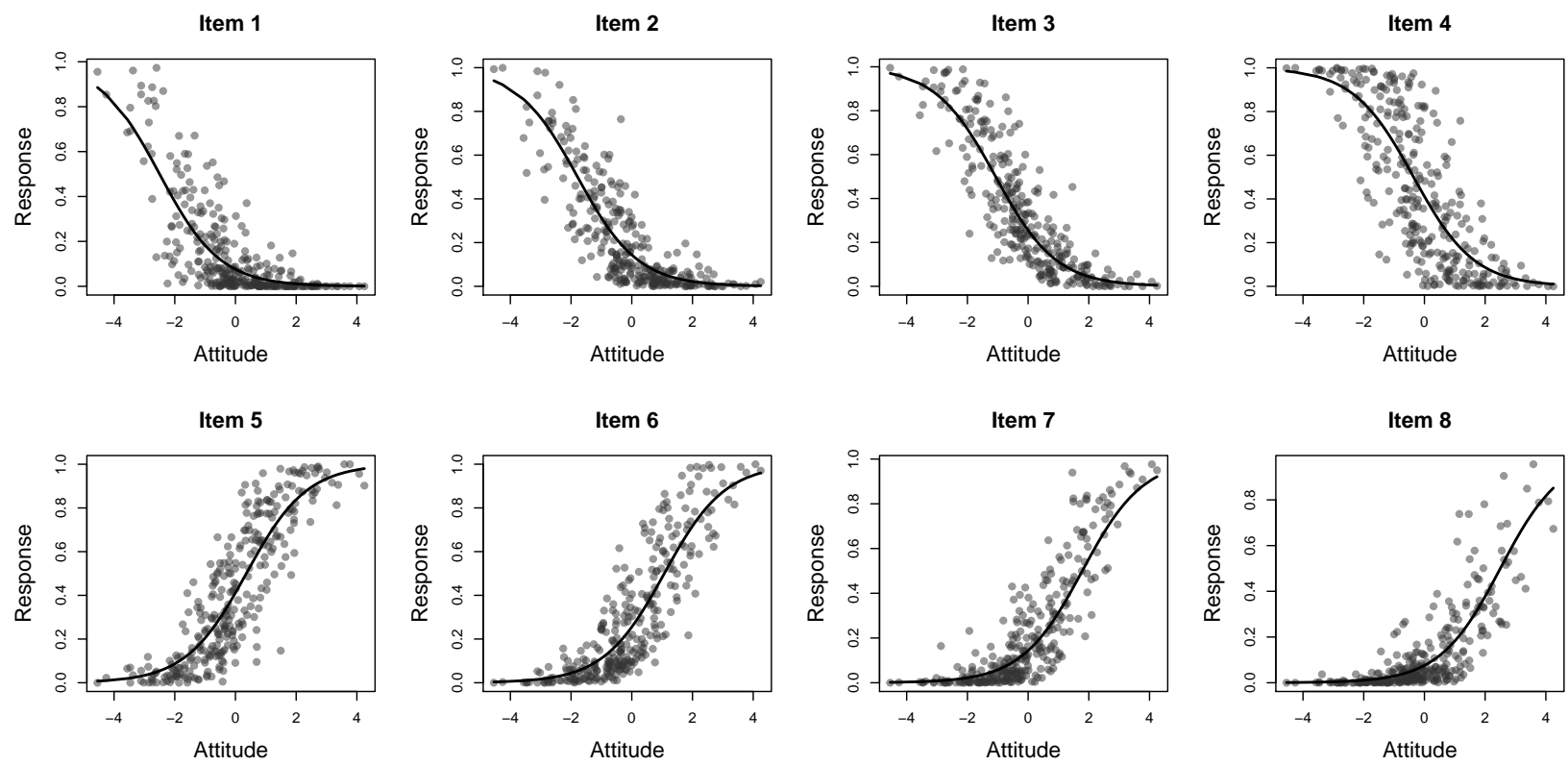

Figure 2. Simulated data from a bipolar Beta Response Model (Noel \& Dauvier, 2007) on 8 sample items (with locations evenly spaced between -2.5 and 2.5 ) half of them with decreasing and half of them with increasing response functions.
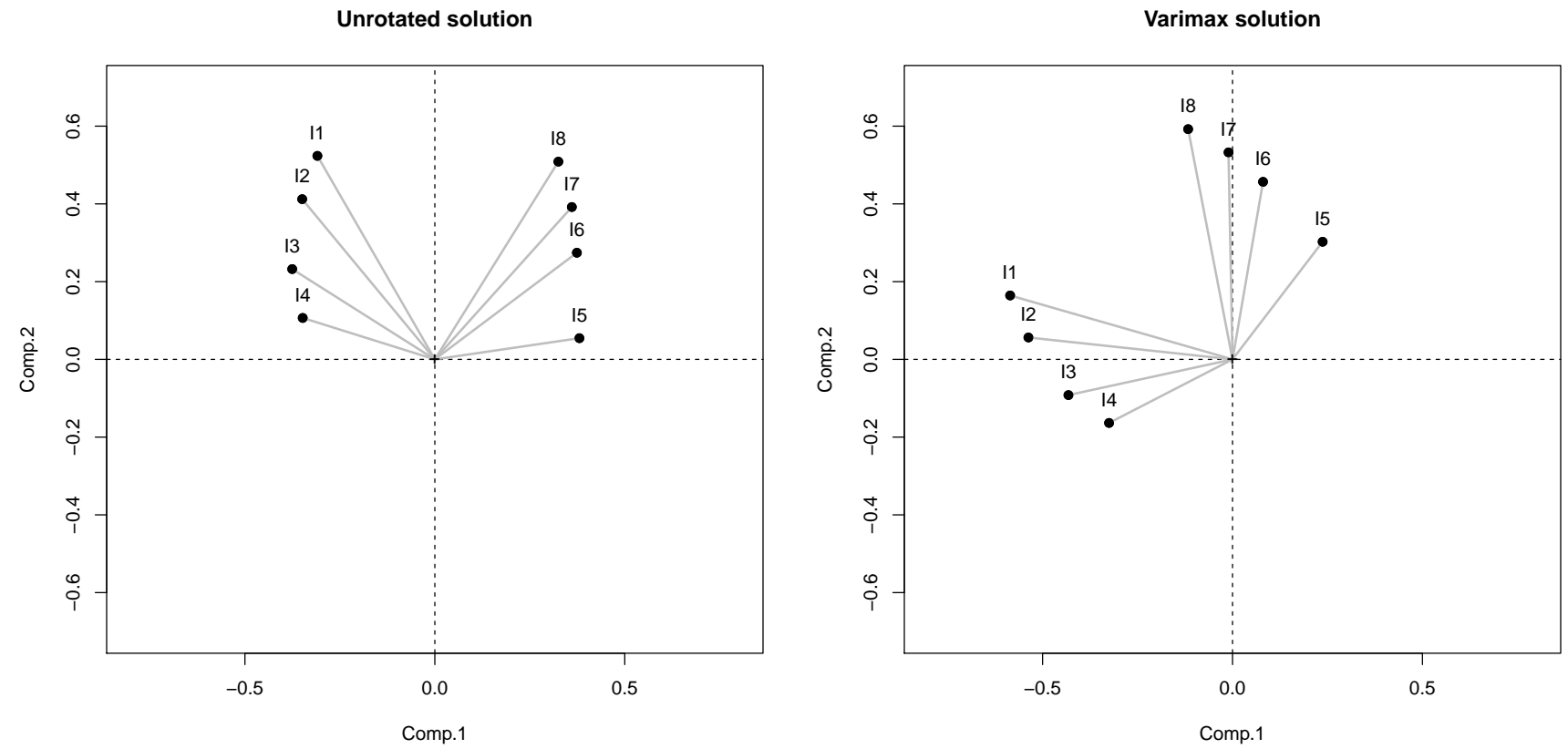

Figure 3. Unrotated (left panel) and varimax rotated (right panel) principal component analysis solutions on a set of simulated items from a bipolar scale. 
ranged in columns in a $N \times p$ matrix $\mathbf{C}$ ), that is the vectors of projections of the observed data vectors (arranged in the $N \times p$ matrix $\mathbf{X}$ ) onto the set of eigenvectors of the correlation matrix (arranged in columns in the $p \times p$ matrix $\mathbf{U}$ ), that is $\mathbf{C}=\mathbf{X U}$. Because $\mathbf{U}$ is of full rank and orthogonal, we can reconstruct the (standardized) observed data matrix by writing:

$$
\mathbf{X}=\mathbf{C U}^{-1}=\mathbf{C} \mathbf{U}^{\prime}
$$

This may be viewed as a regression equation of the observed variables in $\mathbf{X}$ onto the principal components in $\mathbf{C}$ with regression coefficients in $\mathbf{U}^{\prime}$, with this particular constraint that the coefficient vectors have unit norm. One way to understand the meaning of these components is to plot them as a function of the true $\theta$ values, which has been done for our simulated data on Figure 4. This may be viewed as a parameterization of the eigenvectors into eigenfunctions of the true latent attitude $\theta$. Because the data are noisy, principal components extracted from the matrix of true expected responses have been superimposed (as lines on the plot), to make things clearer. What this figure makes appear is that eigenvectors (or projected scores onto them) act very much like a basis of orthogonal polynomials in the reconstruction of the data matrix: The components are first order, second order, third order etc. functions of the latent dimension. Because components extracted from the observed data are very noisy and have low variances (eigenvalues), this is more clearly seen for the true principal components (plotted as plain lines), especially for higher order components. A very similar phenomenon has been pointed out as an explanation of the so-called "horseshoe effects" in correspondence analysis. Benzécri (19731982) showed that in this cases, the $n$-th factor was a $n$-th polynomial transform of the first factor. This is what generally appears when applying correspondence analysis to intrisincally cumulative (or Guttman) scales, not reducible to a linear model.

It is also well-known that PCA is a particular case of a Karhunen-Loève transform that, on a discrete and finite onedimensional signals, automatically finds the best orthogonal basis that minimizes square reconstruction error. As it appears, there is a strong analogy between the common problem in signal theory to characterize the most salient features of a time signal, and the psychometric analysis of response functions: Eigenfunctions of time are looked for in the former case, while eigenfunctions of an unknown latent attitude are looked for in the latter. Although there is no general analytical expression for these eigenfunctions, which depend on the nature of the analyzed signals, an analytic form may sometimes be derived for some a priori known generative processes, such as 1D Markov or Wiener processes (Dony, 2000, p. 10), in which cases sine bases emerge. What PCA does in our case is to find a minimal set of common shape features, shared by all items simultaneously, which provides the best reconstruction of the response functions. Those fac- tors have no psychological meaning, but are purely formal features.

In this line, we may want to observe the quality of data reconstruction (for sample items 1 and 5) by entering an increasing number of components in Equation 7, beginning with the ones having highest eigenvalues. The quality of this reconstruction is illustrated on Figure 5, by entering from 1 to 3 leading principal components from a PCA decomposition on the matrix of the true expected response functions. On each row, the first panel plots the weighted components (principal component times the corresponding loading). This helps visually appreciate the importance of the three first components in the reconstitution of the unknown response functions. We see that the unknown response functions are very well recovered with as few as three components (see last panel of each row on Figure 5). Item 1 has loadings $0.325,0.512$, and 0.521 on the first three components in the true expected response space. For this item with a decreasing response function, the first loading is negative (-.325) which provides a first decreasing shape feature. We see that inclusion of this only component largely underestimates the expected response for extreme subjects (with scores around -4 or +4$)$. This is compensated for by the inclusion of the second (U-shaped) component with a positive loading (.512). The reconstruction is already very good, but still improves with inclusion of the third component, although by a modest amount. For item 5, which is an intermediary item (location .357), the contribution of component 1 is very important, as is visible from the first and second panel of the bottom row $\left(R^{2}=.978\right)$. There is very little difference in the goodness of fit due to the inclusion of components 2 and 3 .

Conclusion. What this analysis reveals is that, although in the case of essentially unidimensional data principal components have a clear mathematical meaning of function shape components, they have no true psychological meaning. In the present case, although the first (unrotated) component correctly reveals a bipolar structure, and models intermediary items (e.g. items 4 and 5) pretty well, we see that what essentially characterizes the second and third components is that they provide the necessary nonlinear adjustment to provide an acceptable fit of the response functions for extreme items. Within the unrotated solution, this would lead the applied researcher to falsely cluster opposite variables into the same factor 2 (see items 1 and 8 on 3, left panel). The use of a Varimax rotation makes things still worse, by gathering subsets of opposite variables into orthogonal factors (Figure 3, right panel). This is one of the sources of this paradoxical result that negatively correlated items appear as orthogonal factors in mood studies. As another consequence, we also note that although the first (unrotated) component correctly recovers the bipolar opposition between both subsets of items, the ordering of loadings is almost reversed within each subset by comparison with the true one. Item 1 for example, which 

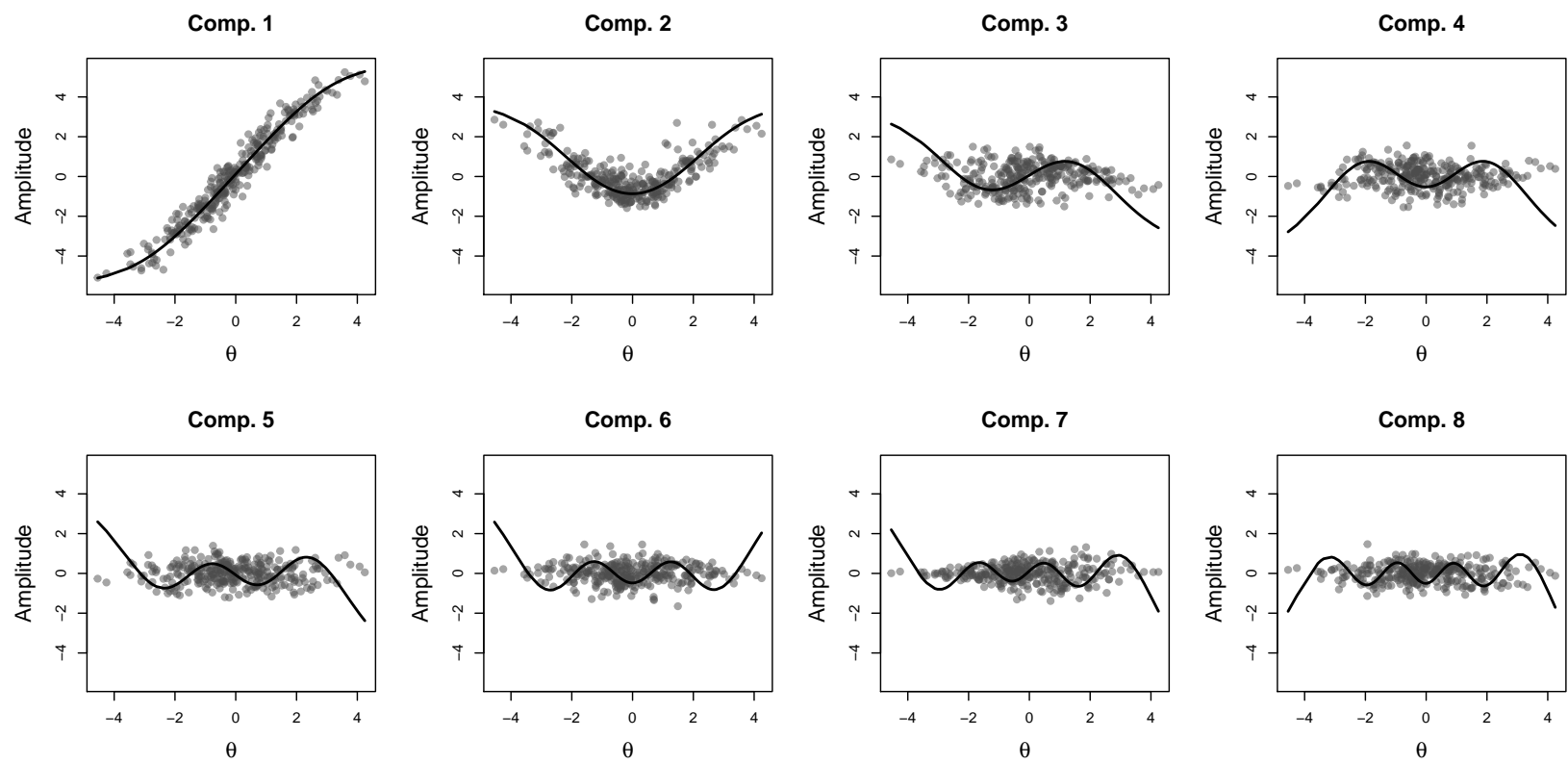

Figure 4. Plot of principal component vectors as a function of the true $\theta$ values, from both the observed data (dots) and the true expected responses functions (lines). Principal components act like orthogonal polynomial transforms of the latent variable.

is the most extreme on the left (Figure 2) has the highest negative loading (Figure 3, left panel).

\section{Study 2: A reanalysis of Noel \& Dauvier (2007) data}

A number of authors have defended the two-factor model of valence, on the basis of factor analysis results (Watson \& Tellegen, 1985), but also on the basis of the psychological argument that positive and negative affect could be experienced at the same time (e.g. Briesemeister, Kuchinke, \& Jacobs, 2012; Cacioppo \& Berntson, 1994). By contrast, others have defended a bipolar model (Russell, 1979, 1980), arguing that it clearly emerged as soon as arousal items were included in the analyses, or invoking methodological arguments to explain why the bipolar structure did not always so clearly emerge (Green, Goldman, \& Salovey, 1993).

Data. We reanalyze here in a new way Noel and Dauvier (2007)'s dataset, consisting of 19 mood valence items rated by 464 persons. This set was constructed by adding to an initial set of mood adjectives, some items from the Hospital Anxiety Depression Scale (Zigmond \& Snaith, 1983), to include descriptors of extreme negative states. Items short names are: "Tense-woundup", "Frightened", "Worried", "Butterflies", "Panic", "Unhappy", "Sad", "Nervous", "Annoyed", "EnjoyThings", "Laugh", "Cheerful", "LookForwardEnjoyment", "EnjoyGoodBook", "Content", "Elated", "Happy", "Pleased", and "Euphoric". Although an extended model, with better properties, will be presented in the next sections, Noel and Dauvier (2007) showed that a unidimensional bipolar Beta Response Model already provided an ac- ceptable fit to the data, with a clear scaling of items from a negative to a positive end of a valence dimension, thus supporting the bipolar view. So, we are interested in looking at these data with factor techniques (traditional standardized PCA will be used), with and without additional varimax rotation.

Statistical analysis. Although parallel analysis (Horn, 1965) suggests to retain three components, with eigenvalues 7.31, 2.06 and 1.43 (unrotated solution), PCA solutions with and without Varimax rotation in the first two factor space are first examined (loading plots are displayed on Figure 6), for illustration and discussion purposes. Both solutions lead to very different interpretations: The unrotated solution strongly suggests a bipolar negative vs. positive affect model on the first factor (although the second factor has nonnegligible loadings), and the rotated solution more strongly supports a two-factor solution, with a negative and a positive affect factors.

But returning to the raw data and examining subjects factor scores on the first three principal components of an unscaled PCA (displayed as dots in a 3D plot on Figure 7, left panel), it appears that the observations follow a curvilinear pattern in the multivariate response space (and this shows itself even more clearly when using specialized software for 3D dynamic rotation). This means that scores on one factor are in some way non-linearly related to scores on the others, so that there is a strong suspicion that the three factors are actually not independent and should not be interpreted separately. In this context, and in the line of the arguments of the previous section, a principal curve (not component) passing 

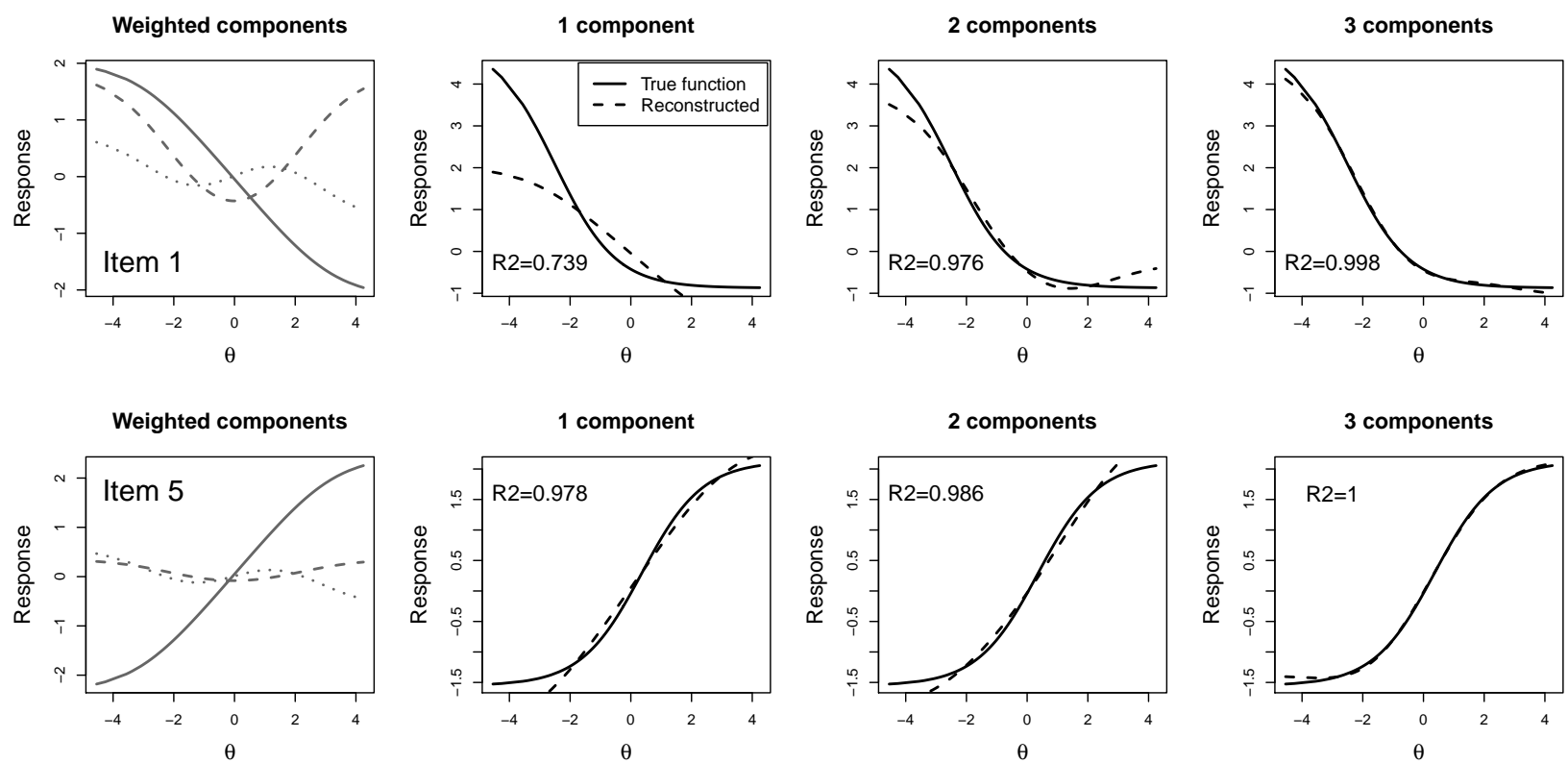

Figure 5. Reconstruction of the standardized response functions of items 1 and 5 by adding from 1 to 3 principal components in the reconstruction formula. On each row, the first panel gives the weighted components (principal component times the corresponding loading) and the following panels show the cumulative addition of these weighted components.
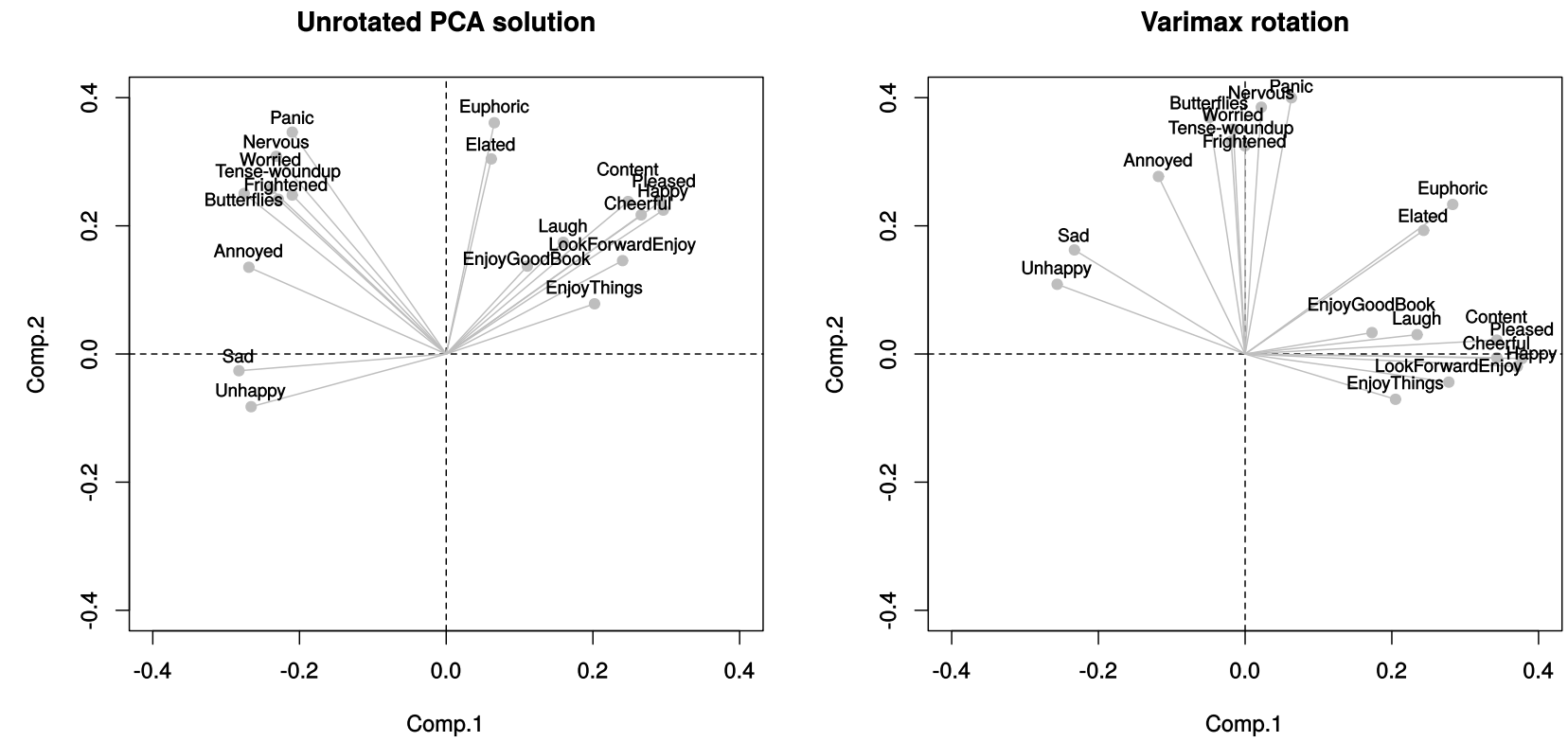

Figure 6. Principal component analysis of 19 mood items, with and without varimax rotation in the space of the two first factors. 
through the middle of the data (in some sense) is looked for.

A principal curve in $p$-dimensional space is defined as a smooth (infinitely differentiable) parameterized curve $\mathbf{f}(t)=$ $\left(f_{1}(t), \ldots, f_{p}(t)\right)$ that $\left.i\right)$ has finite length inside any bounded subset of $\mathbb{R}^{p}$, ii) does not intersect itself and iii) is selfconsistent, which means that each point on the curve is the conditional mean of the observations projecting on $\mathbf{f}$ around this point (Hastie \& Stuetzle, 1989). This is exactly the situation we are facing when analyzing multivariate response vectors generated through a set of $p$ unknown response functions $f_{1}, \ldots, f_{p}$ for $p$ items in a questionnaire. A Principal Curve Analysis has been performed on these data (using the R princurve package, Hastie \& Weingessel, 2013).

Results. The resulting flexible curve, providing an approximation to the multivariate expected response, has been added to the $3 \mathrm{D}$ observation plot (Figure 7, left panel). This curve is here obtained in a non parameterized form using a LOWESS smoother. Orthogonal projections onto the curve are used to provide subjects scores, computed as curvilinear distances of this projection from one extremity of the curve. Interestingly, the correlation between these scores and the latent scores estimated from a Beta Response Model is $R=0.93$ (see Figure 7, right panel). This both provides support for Noel \& Dauvier (2007) parametric model, for this set of items, and shows that Principal Curve Analysis does a good job at "unfolding" the between item curvilinear relationships. Principal curve analysis allows estimating latent scores in a non-parametric way, with no prior assumption neither on the response function nor on the response distribution.

Once estimated, scores and smoothed response function may be inspected to better understand the nature of the nonlinearity. The estimated response functions may be examined by plotting the smoothed responses on a given item (i.e. the corresponding marginal curve) against estimated curvilinear scores. Figure 8 displays these estimated response functions for a selection of four negative (Unhappy, Sad, Worried, Nervous) and four positive (Cheerful, Pleased, Content, Happy) affect items. All negative items show a monotonic decreasing response function and all positive items a monotonic increasing one, in good concordance with Noel \& Dauvier (2007) results.

Conclusions. These results strongly support a bipolar model of emotional valence. Though floor (Unhappy, Sad) and ceiling (Worried, Nervous) effects are perceptible on these plots, one may note that the non-linearity is not so pronounced, and that a linear model would probably give an acceptable approximation. This is why the unrotated PCA solution already provided a good representation of the bipolar dimension. But although modest, this slight curvature at the item level is sufficient to create a bended multivariate data cloud at the whole data set level, as seen on Figure 7 (left panel), and this in itself is sufficient to induce an inflation in the number of factors retained in a PCA by common criteria. Moreover, a varimax rotation in this context would lead to retain factors (actually portions or branches of a curve, see Figure 1c) with no real (or incidental) psychological significance.

Although it has some limitations of its own (in particular a smoothing parameter need to be arbitrarily set), principal curve analysis may thus be a useful diagnostic of nonlinearity, and in many cases a preliminary exploratory step to a more formal analysis using a well defined parametric response model. In this context, fitting a Beta Response Model is nothing more than looking for a fully parameterized principal curve (based on a logistic response function) in the multivariate response space, with a proper distributional model (a Beta distribution) that takes into account the bounded nature of the data.

\section{The Heterogeneous Unfolding Model of Emotion}

\section{On a nonlinear dynamic relationship between valence and arousal}

Arousal has been voluntarily set aside in the previous discussion, to make clear that the extra factor phenomenon could not be attributed to the specific contribution of an arousal component of emotion. Arousal is reintroduced in this section, with a specific hypothesis on its relationship to valence.

Let us consider an organism in a positive stable mood state $P$ (see Figure 9, on the right hand side) and let us consider that this organism is submitted to a strong negative stressor from the environment. The hypothesis to be examined in this section asserts that, in conjunction with an increase in negative feelings (or a decrease of positive mood), the arousal level will increase, up to some state $A$ (center of Figure 9), arguably because the organism has to react against the stressor or control it, and protect itself. Then two scenarios may be imagined: Either the control is effective and the organism returns to the positive stable state $P$ (a movement hereafter named positive deactivation), or the control attempt fails and beyond some point of time and energy spent to no avail, the organism may give up fighting and fall into a depressive state $N$ (negative deactivation), as is well known from learned helplessness studies (Seligman, 1972). In both cases, state $A$ is temporary and unstable, as the organism seeks tension reduction. Put together, both assumptions lead to a model of affect where arousal varies as a single-peaked function of valence, conceived of as a bipolar (positive-negative) dimension, the more active states lying in the middle area. That state $P$ is a "homebase" and stable state is supported by empirical evidence showing a tendency to avoid negative stimuli but also high levels of arousal (Robinson et al., 2004). To a lesser extent, this leads to expect that the depressive deactivated $N$ state may also show some stability, as the loss of 

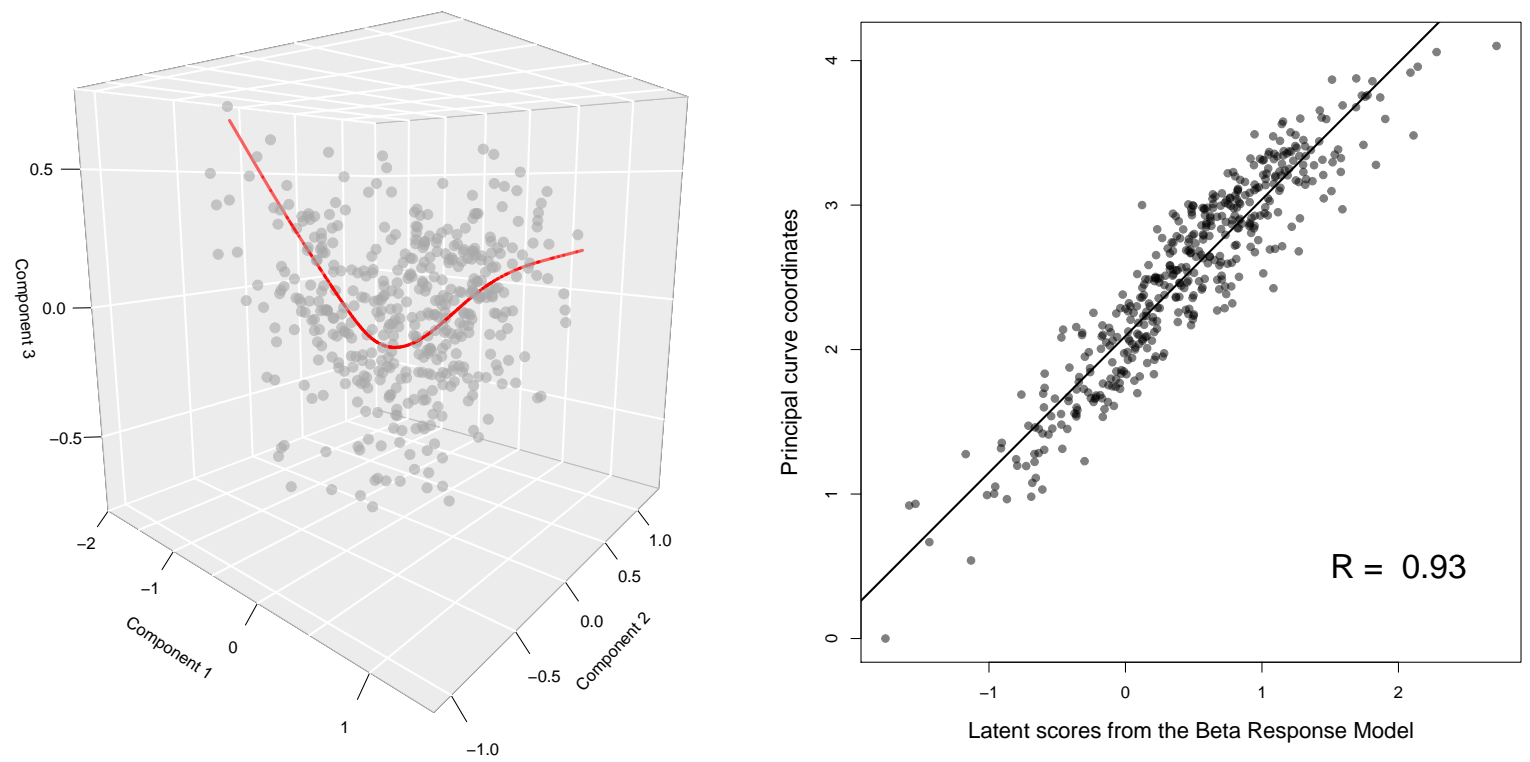

Figure 7. Left panel: Principal curve in the 3D PCA observation plot of Noel \& Dauvier (2007) mood data. Right panel: Correspondence between curvilinear scores on the principal curve and latent scores recovered from a Beta Response Model fit.
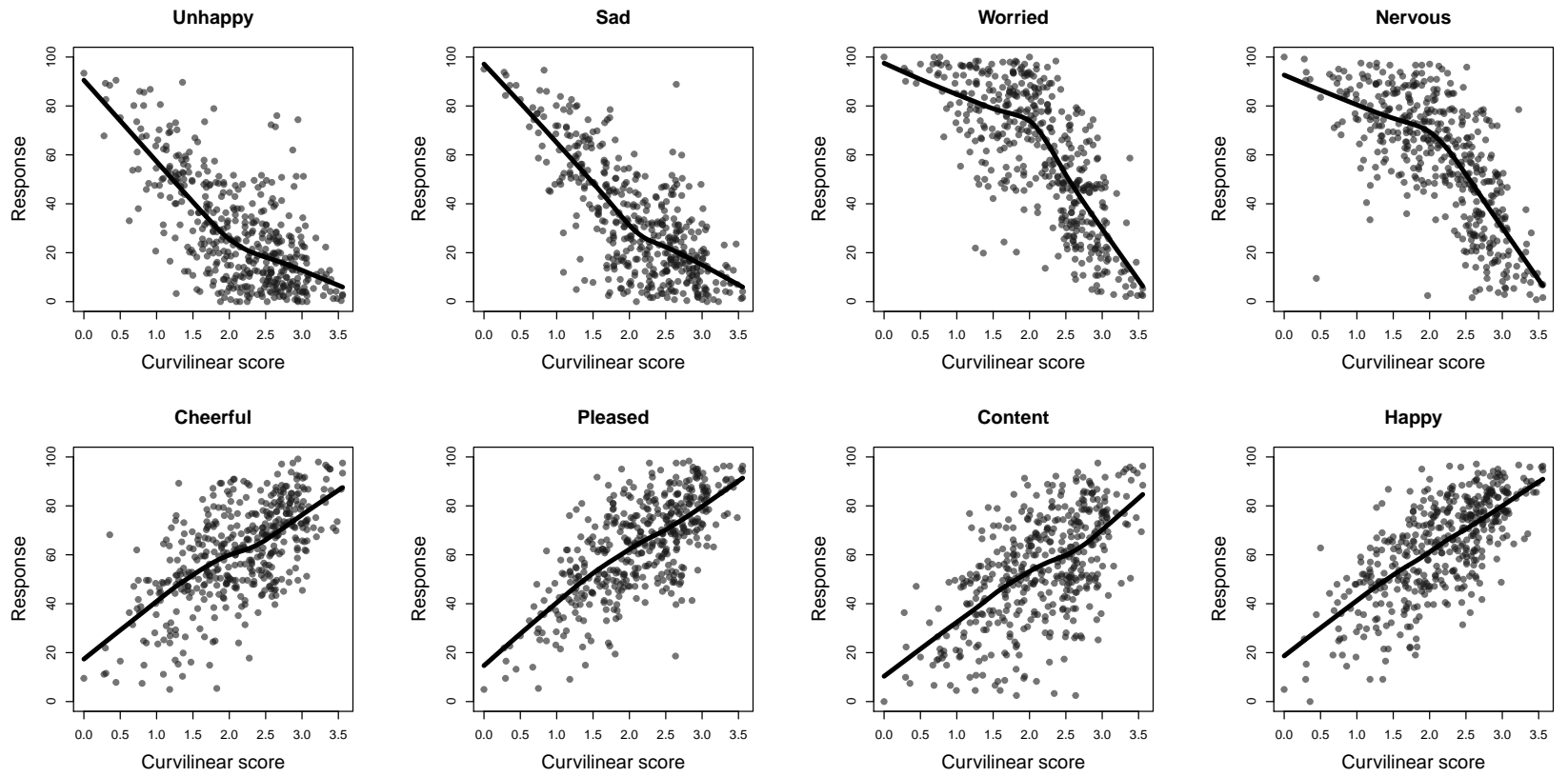

Figure 8. Scores and response functions as estimated by Principal Curve Analysis for 6 affect items.

energy may render it difficult to restore a positive mood state.

If this model is correct, then there are some specific expectations that may be stated as to the shape of response functions to emotional items. If the axes are arbitrarily oriented as on Figure 9 (with positive valence on the right hand side), then an item like "Serene" is expected to have a monotone increasing response function, and an item like "Sad" a mono- tone decreasing response function, with respect to some unknown mood value $\theta$ along this dimension. Moving rightwards, response to both items are expected to respectively, increase and decrease. But items related to arousal (like "Activated" or "Stimulated") are expected to have an invertedU-shaped response function. Conversely, low arousal items (e.g. "passive", "sleepy"), as opposite of active items, are 


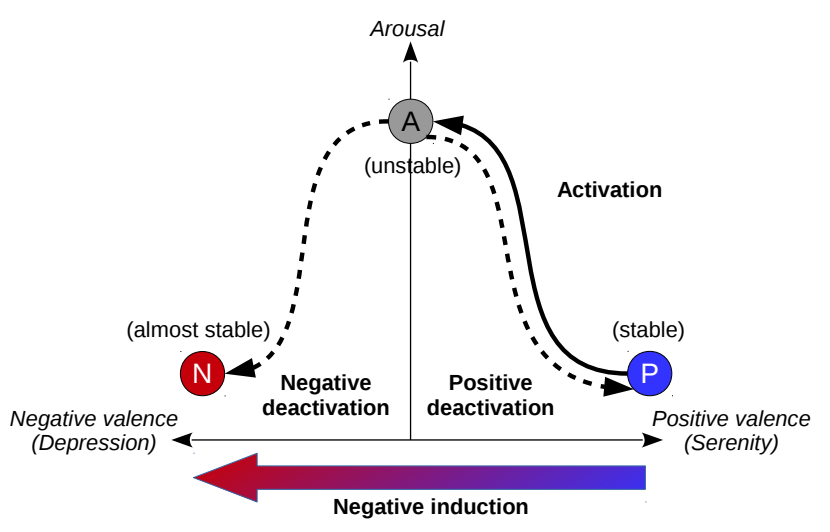

Figure 9. An hypothetical activation-deactivation mechanism. Upon negative constraint from the environment, the organism increases its arousal (alertness). Effective control of the environmental stressor results in positive deactivation, and ineffective control results in negative deactivation (depression or learned helplessness).

expected to display an inverted pattern of response, that is a U-shaped response function. These expectations for the basic positive, negative, passive and active emotional states are graphically displayed on Figure 10 (left panel) on an arbitrary $[0 ; 100]$ response scale.

A specialized item response model for analyzing unimodal response functions is known under the name "unfolding model" in Item Response Theory, after Coombs (1950). This name comes from the fact that if an inverted-U shaped response function does underlie the data but is unknown, then traditional monotone or linear scaling techniques (factor analysis, PCA, multidimensional scaling) will simply scale observations from low to high responses, thus blindly folding the true latent dimension. In this situation, if data were available on a single item, then unfolding would be impossible. But collecting responses on several items makes it possible to recover scores on the latent dimension. This model has been shown to be well-suited to the modeling of ambivalent attitudes (Andrich \& Luo, 1993; Noel, 2014; Roberts, Donoghue, \& Laughlin, 2000) or in the modeling of behavior change, when processes of change follow an activationdeactivation time pattern (Noel, 1999). Up to our knowledge, only one study (Van Schuur \& Kruijtbosch, 1995) considered unfolding as a candidate model for affect items, and obtained very good results on binary data, but at the prize of excluding deactivation items. A particularity of emotion data, from an unfolding perspective, is the presence of inverted (or low arousal) items, leading to U-shaped (and not only invertedU-shaped) response functions. Although this case had not been previously described in the unfolding literature, it is easily dealt with in practice by simply inverting the corre- sponding items, before applying a common unfolding model, as will be illustrated in the last section of this paper. Within the proposed model of emotion, combinations between extreme valence states and high level of arousal (e.g. anxiety or exaltation) are expected to also have an inverted-U-shaped response function but located in the middle left area for the former, and in the middle right area for the latter (see Figure 10, right panel). This model will be named the Heterogeneous Unfolding Model of Emotion (HUME), where the word "heterogeneous" represent between and within subject variability (to be justified in study 4 below).

As can be seen on the figure, the HUME response functions could artificially be viewed as portions of sine or cosine functions (a fully specified parametric form will be given below). So the model predicts that factor and principal component analyses will result in circular patterns of points. More precisely, correlations between items that have relatively close response functions (e.g. Depressed and Stressed or Serene and Calm, in the examples of Figure 10) will be high, and then lower and near 0 as more distant items are considered (e.g. Depressed and Active), and then high again but negative when very distant (opposite) items are examined (e.g. Depressed and Excited or Depressed and Serene). But this geometric effect of a seemingly sinusoid variation is purely superficial as there is no serious theoretical reason to expect that, say, Serenity, will ever decrease as one moves further towards $+\infty$ on the valence dimension. Although somewhat insightful from a graphical point of view, the circumplex representation falsely conveys a cyclical hypothesis that is not psychologically nor biologically reasonable. Note that, from the previous arguments, the HUME also paradoxically predicts that some non-negligible correlation will exist between items such as Excited and Calm, because a monotonic relationship exists between both response functions on at least a portion of the latent dimension (approximately between -1.5 and 1.5 on 10, right panel). A standard PCA performed on these theoretically generated response curves is displayed on Figure 11, where both Excited and Calm items show a positive loading on the first factor.

The HUME model has important differences with previous proposals. First, the valence dimension is neither a positive versus negative emotionality dimension, in Tellegen et al. (1999) terminology, nor a pleasantness-unpleasantness bipolar dimension, in the sense of Barrett and Russell (1999). Positive and negative affect have been associated with high levels of arousal by the first authors, and medium levels of arousal by the last ones, while they are associated with low levels of arousal in the present proposal. Second, this model is clearly a bipolar model of mood, in the line of Russell (1980), but in this formulation, it can be seen on Figure 10 that a bipolar model is not contradictory with mixed feelings, an argument often put forward against bipolarity (Briesemeister et al., 2012; Larsen \& McGraw, 2011; Larsen, Mc- 
The basic HUME model

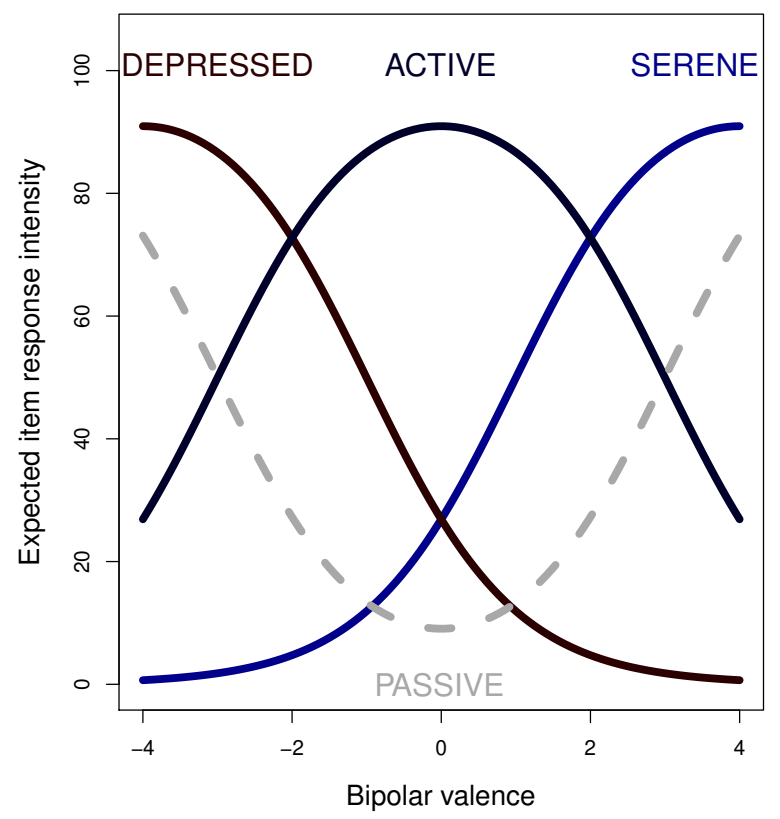

Combined and opposite states

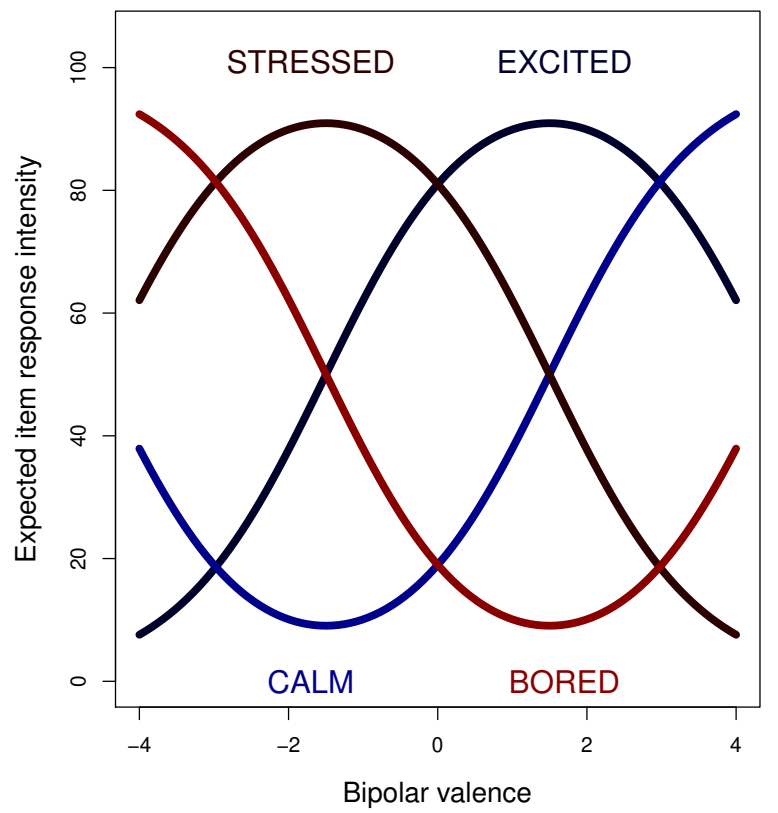

Figure 10. The HUME model of affect. Left panel: Basic states. Right panel: Composite emotions.

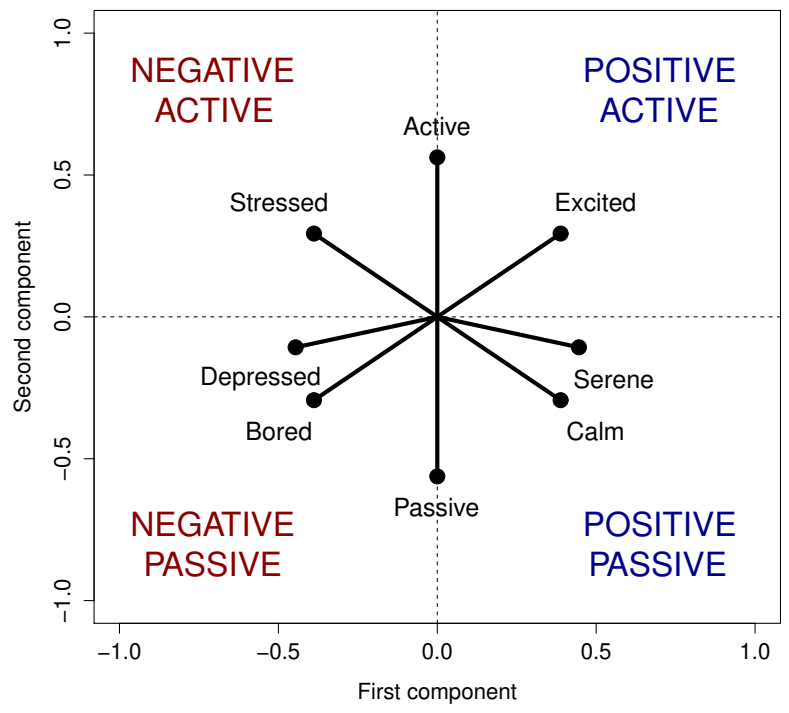

Figure 11. The circumplex structure of emotion as resulting from a principal component analysis on the HUME theoretical response functions.

Graw, \& Cacioppo, 2001). A person may well positively respond on both Anxious and Excited items (at medium levels), or even Depressed and Serene (at low levels), but the model predicts that it cannot be at high levels of intensity (see the crossing points of each pair of curves on Figure 10), and that upon moving along the dimension in either direction, one aspect (positive vs. negative) will eventually dominate. Third, the model allows representing opposition relationships between emotions as various symmetry operations on the response functions. A horizontal flip of a given response function along the valence dimension gives the response function of a valence-opposite item (e.g. Anxious-Excited). But a vertical flip yields that of a fully opposite item, i.e that would appear as the symmetric point about $(0,0)$ in a circumplex representation (e.g. Anxious-Calm). Combining a vertical and a horizontal flip gives an activation-opposite item (e.g. Excited-Calm). In this context, the inverted-U shape hypothesis also leads to unusual predictions. For example, Excited and Bored have been often found to appear as full opposite in a circumplex representation (Russell, 1980; Russell, Lewicka, \& Niit, 1989). So assuming an invertedU-shaped response function for Excited implies expecting a symmetric U-shaped response function for Bored (Figure 10 , right panel) with the important theoretical consequence that higher responses to Bored are expected on the negative side (at high levels), but also on the positive side (at low to medium levels). Although unusual, this prediction makes sense: A risk of a state totally devoid of stress may well be under-stimulation and boredom. Fourth, in this presentation, arousal can no more be conceived of as an intensity dimension for negative and positive feelings, at least not in a usual dimensional representation, as high levels of arousal 
are expected to occur in the middle of the valence dimension.

\section{Study 3: A reanalysis of the International Affective Pic- ture System dataset}

Data. The HUME model also leads to very different theoretical expectations than the ones deduced from a model recently proposed by Kuppens et al. (2013). These authors directly addressed the same question of a nonlinear relationship between arousal and valence, by systematically comparing constant, linear, $\mathrm{V}$-shaped and inverted-U shaped models of relationship. They provide empirical evidence in favor of a V-shaped model, in concordance with what had been previously reported by Lang, Bradley and colleagues, based on averaged ratings of Pleasure and Arousal evoked by affectively laden images from the International Affective Picture System (IAPS) (Lang et al., 2008). This publicly available dataset is reanalyzed here, in the light of the HUME assumptions, along with averaged ratings reported by Mikels et al. (2005) on a number of elicited feelings (Anger, Disgust, Fear, Sadness, Awe, Contentment and Excitement), for a significant subset of the IAPS pictures.

Hypothesis. In the V-shape model, high levels of activation are expected for extreme (negative and positive) valence states. Activation may then be considered as practically synonymous to (positive or negative) emotion intensity. Under the HUME, extreme valence states are associated with low levels of activation, and high levels of arousal are associated with mixed feelings or relative affect neutrality.

We may note that, though supported by an important amount of empirical data, the V-shape hypothesis leads to at least three counterintuitive predictions, as noted by Kuppens et al. (2013) themselves. It predicts that states of i) high pleasure and low activation, ii) intensely unpleasant and low activation, and iii) neutral or intermediary valenced states of high activation, cannot occur. But obviously serenity, depression and surprise are acceptable examples of these three cases. On these three points, the HUME hypothesis leads to more realistic predictions.

To understand the source of this apparent contradiction between both models, it must be underlined that standard instructions given to participants in an IAPS study are to rate pictures in terms of Pleasure, Arousal and Dominance on a bipolar 9-point pictogram response scale named SAM, for Self-Assessment Manikin (Bradley \& Lang, 1994). As to pleasure, the pictogram response scale range from a smiling happy figure to a frowning unhappy figure. This response format actually induces a definition of valence where extreme positive and negative states are fairly active. Within the HUME, such affect states are not the most extreme of valence, and should, like arousal, display an inverted-U-shaped response function (Figure 12, left panel). If this holds true, then this should show itself in a curvilinear ("rabbit ear") pattern on a correlogram plot of Arousal against Pleasure (or Displeasure). This prediction is illustrated on Figure 12 (right panel). Because the SAM does not separate responses on Pleasure and Displeasure, and chooses to oppose them on a bipolar format, the Displeasure-Arousal theoretical space curve must be horizontally flipped from right to left to get the whole relationship pattern that is expected from the HUME, in this constrained response space. Clearly, this pattern should appear as two "rabbit ears".

Data analysis. This expected pattern was compared to the bivariate plot of the publicly available IAPS norms (Lang et al., 2008) on valence and activation (Figure 13, left panel). At first glance, it clearly displays what has been described as a "boomerang" or V-shaped characteristic pattern.

To test the HUME predictions of inverted-U shaped response functions, we performed unfolding analyses, using the Beta Unfolding Model (Noel, 2014). The Beta Unfolding Model (BUM) allows simultaneous scaling of persons and items on a single dimension when responses are expected to follow the kind of single-peaked pattern described in the previous sections. It is especially designed to deal with bounded responses, by assuming a Beta distribution, and a specific response function. Within the BUM, the expected response function for person $i$ (with latent attitude $\theta_{i}$ ) to item $j$ (with ideal location $\delta_{j}$ ) reads:

$$
E\left(X_{i j} \mid \theta_{i}\right)=\frac{\exp \lambda_{j}}{\exp \lambda_{j}+\exp \left[\left(\theta_{i}-\delta_{j}\right)+\tau_{j}\right]+\exp \left[-\left(\theta_{i}-\delta_{j}\right)+\tau_{j}\right]}
$$

Beside a specific location $\delta_{j}$ (i.e. that point on the valence continuum where a particular emotion reaches its peak level), each emotional item is characterized by an acceptability parameter $\lambda_{j}$ (which controls the amplitude of the response curve) and a precision (or inverse dispersion) parameter $\tau_{j}$ (which controls the dispersion of responses around the curve).

Because Pleasure and Displeasure were not rated separately in the original study, but instead rated on a single "Valence" bipolar 1-9 scale (from Displeasure to Pleasure), separate ratings of Pleasure and Displeasure were reconstructed by splitting the response scale in two parts. All ratings in the 5-9 range were considered Pleasure ratings, and low Valence ratings (in the $1-5$ range), properly inverted $\left(x^{\prime}=5-x\right)$, were considered as measures of Displeasure intensity (note that, because the available data are averaged ratings, no rating is exactly 5). Two separate unfolding analyses were then performed, on the subset of positively valenced pictures (with Pleasure and Arousal as items) and then on the subset of negatively valenced pictures (with Displeasure and Arousal as items). Because two items may be too few for a stable unfolding analysis, a complementary analysis integrated the averaged ratings on Anger, Disgust, Fear, Sadness, Awe, Contentment and Excitement, reported by Mikels et al. (2005).

Results. Inclusion of Mikels et al. (2005)'s data resulted in a slightly better fit (although the results from the two-item 
HUME response functions

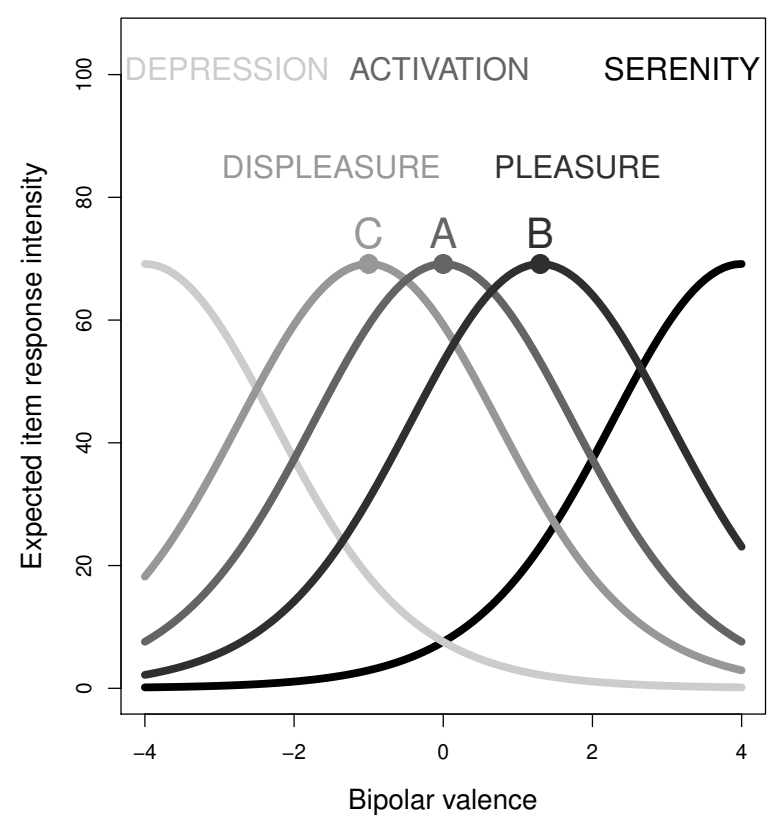

Expected relationships

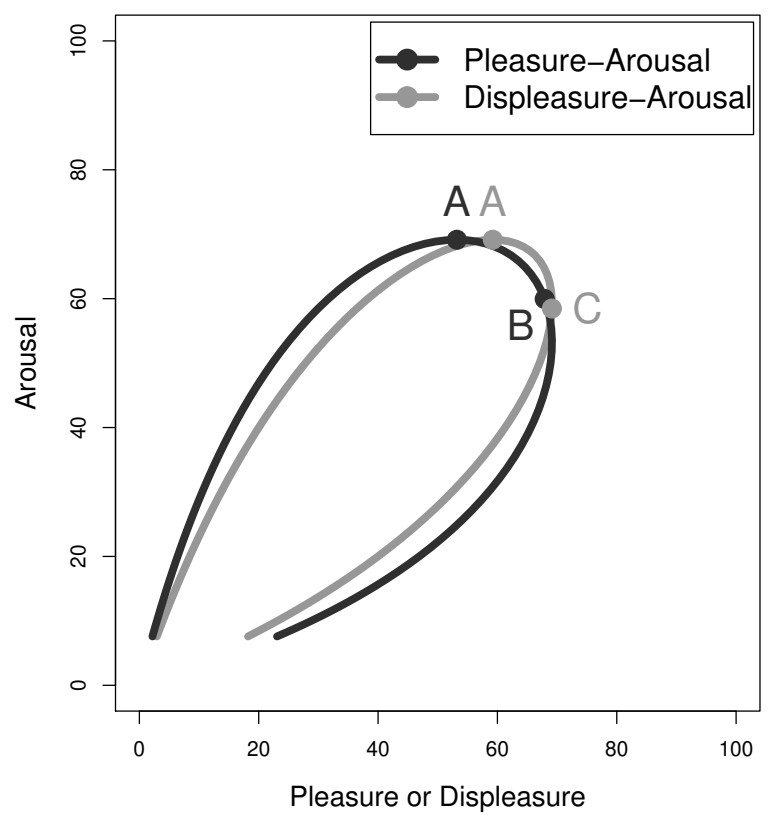

Figure 12. Expected response curves (left panel) and expected bivariate relationships between Pleasure (or Displeasure) and Arousal (right panel) as deduced from the HUME model.

analyses were indeed very close) and the results reported here are from this second analysis. For both subsets of positively and negatively valenced pictures, the analysis led to estimated responses functions, from which expected correlograms of Arousal against Pleasure and Displeasure were derived. These are plotted as two space curves on Figure 13 (right panel).

In the light of this analysis, the previously reported boomerang shape actually appears as the conjunction of two strongly folded curves, an artifact induced by the constrained Pleasure-Displeasure response format. Examination of some of the sample IAPS picture contents (left panel) strongly suggests that, starting from a bottom center point and going rightwards along the right curve, picture content evolves from relatively neutral content (e.g. "Lamp", "Window", "Plate"), to positive active content (e.g. "Hockey", "SkyDivers"), and then to positive passive content (e.g. "Sunset", "Clouds", "Flowers"). Similarly, on the left hand side, going leftwards along the left curve, content evolves from neutral to negative active ("War Victim", "Mutilation"), and then to negative passive or depressed content (e.g. "Disabled Child", "Cemetery"), in good concordance with the predictions of the HUME.

Mikels et al. (2005) have characterized IAPS pictures by dominant features (among Anger, Disgust, Fear, Sadness, Awe, Contentment and Excitement), based on the more highly rated ones. The averaged locations for each picture category are plotted as category labels on right panel of Figure 13. Once properly unfolded, the latent scaling of these seven categories appears as: Sadness, Disgust, Fear, Excitement, Awe, Amusement and Contentment, once again in good concordance with the HUME prediction that, along a negative to positive continuum, the most active categories lie in the middle range.

\section{Study 4: A test-retest study on emotional change}

As suggested in the previous study, emotion ratings need to be collected separately on monopolar response scales, in order to test the HUME main assumptions. In this line, an empirical study was conducted in a university exam situation, where students were asked to rate their emotional state on a number of items, before and after the exam. This situation was chosen because it is expected to naturally induce emotional change, from pre to post exam time, potentially in both directions (from stress to positive relief, or from stress to sadness), among students. So a number of analyses are possible, on the structure of pre-exam feelings, on the structure of post-exam feelings, and on the correspondence between both.

Subjects. A total of $N=746$ undergraduate students, in Psychology $\left(N_{1}=169\right)$ and Sport Science $\left(N_{2}=577\right)$, were asked to rate a basic set of mood items at the beginning and the end of a one-hour exam. The exams were chosen from our experience in both departments, as those that 

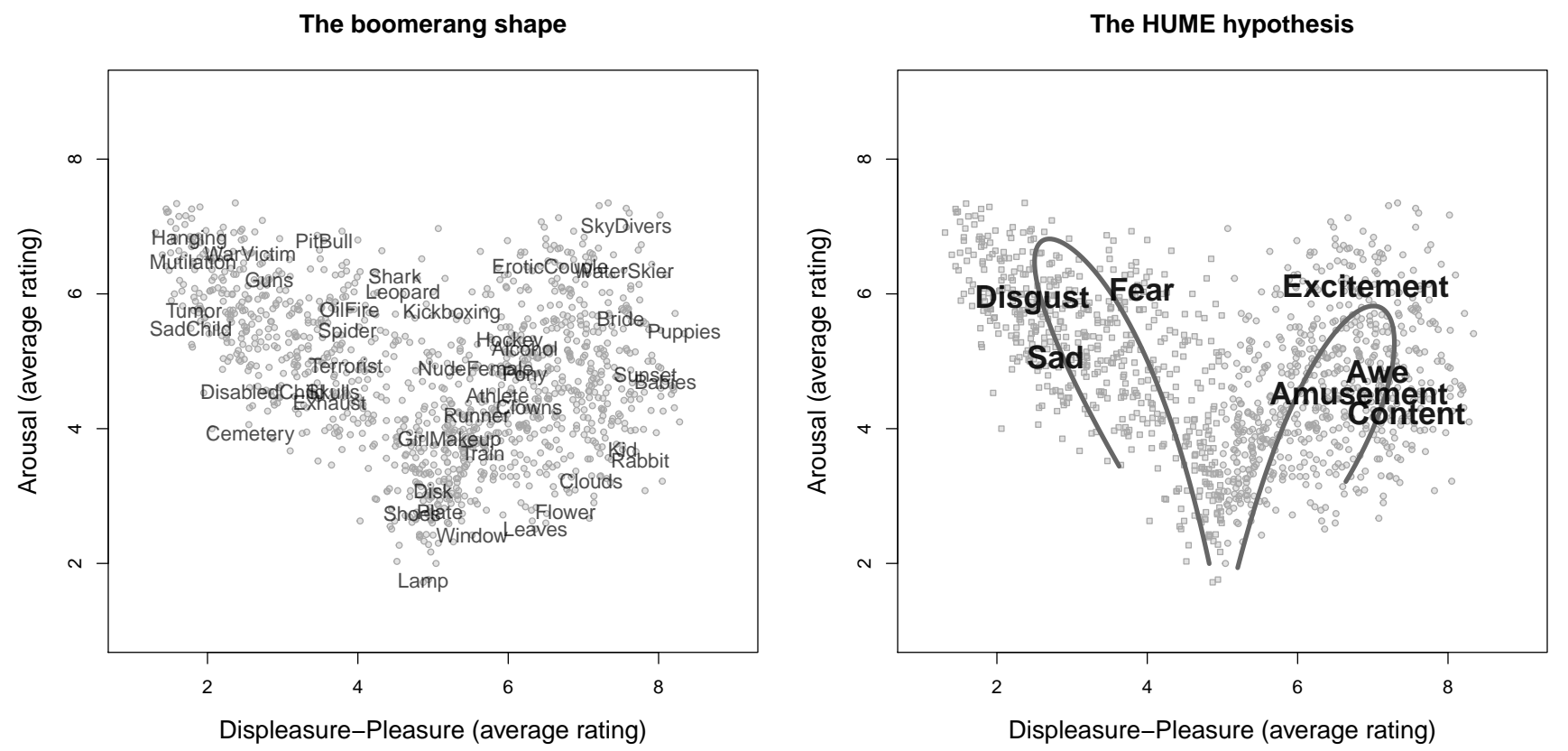

Figure 13. Left panel: Observed "boomerang" pattern of relationship between Pleasure and Arousal average ratings on the IAPS (Lang et al., 2008). Right panel: Predicted relationships between Displeasure and Arousal (left curve) and Pleasure and Arousal (right curve) from the HUME hypothesis. Emotion categories from Mikels et al. (2005) have been added on right panel for interpretation purposes.

students considered as the most stressful (Statistics for psychology students, and Developmental Psychology for Sport students). Because the study took place in an exam setting, no other personal information (age, gender) was collected.

Measures. A subset of 16 mood adjectives was taken from Russell (1980), so that most aspects (i.e. active and passive, positive and negative content) of the classical circumplex of emotions be represented. The adjectives were: Happy, Nervous, Bored, Pleased, Cheerful, Stressed, Tired, Stimulated, Upset, Calm, Alert, Sad, Relaxed, Tense, Depressed, Serene. Because the exam was web-based, students were presented the emotion questionnaire form before the exam form itself. They were told that a study on concentration among students during exams was being conducted, and were asked, as part of it, to rate each of the 16 adjective on a continuous $0-100 \%$ agreement visual analogue scale. All items were presented on the same page, and response scales were 480px long sliders. Items were randomly ordered on the page for each student, using an appropriate PHP/Javascript program. Upon filling the questionnaire, students were redirected to the exam form, and then to the emotion questionnaire again, after the one-hour test. All responses were automatically saved on the web server.

Models and hypotheses. A Beta Unfolding Model (BUM) was fitted to the data, passivity items being properly inverted so that a standard unfolding software can be used. In the context of pre-test post-test analysis, three variants of the BUM were fitted, from the least to the more constrained:

1. A heterogeneous model with separate item parameter estimation for the pre and post exam ratings: This is a reference model against which more constrained hypotheses are tested.

2. A heterogeneous model where emotion peak locations are constrained to be the same from one time to the other, but amplitudes left free to vary. This model asserts a common and stable structure of emotion peak locations on the valence dimension, but allows for changing amplitudes, depending upon measurement time, thus implementing a distinction between the basic valence (i.e. location) of a given emotion, and the emotional response amplitude.

3. A homogeneous model on the full dataset collapsing pre and post exam measures. Within this model, all item parameters are the same for pre and post exam measurements, and only person parameters (i.e. emotional states) are estimated separately at both measurement points (as if they were from different people). This model corresponds to a hypothesis stating that subjects' emotional change is entirely reducible to a simple movement along the valence dimension, response intensities on each item strictly varying following the BUM parametric model 8, up to common parameters estimates.

In the line of the HUME assumptions, it was hypothesized that:

1. Responses can be modeled with a Beta unidimensional 
unfolding model (BUM), with extreme negative and positive mood items showing monotone (respectively decreasing or increasing) response functions, activation items showing inverted-U-shaped response functions, and passivity items displaying $\mathrm{U}$-shaped response functions.

2. If the representation of a stable Negative vs. Positive Valence latent dimension, as described by the HUME, is correct, then separate analyses on students' responses before and after the exam should reveal an identical structure, as far as emotion item peak locations are concerned.

Results. The Akaike Information Criterion was used to compare models (the lower the AIC the better the model likelihood and parsimony). Within each model, INFIT and OUTFIT item fit statistics (Wright \& Masters, 1982), measuring $\chi^{2}$ departure between responses and model prediction, were used to judge adequacy of the parametric response model (values between $[0.6 ; 1.4]$ are usually considered acceptable). The AIC of the three models were -15939.54 (unconstrained heterogeneous model), -15976.28 (constrained location heterogeneous model) and -15288.53 (homogeneous model), indicating that the second model fitted better. Within this model, all item INFIT and OUTFIT statistics were comprised between 0.617 and 1.017, thus lying in the acceptable range. Hypothesis 1 of an unfolding model is thus supported, with peak locations remaining unchanged under mood variation (Hypothesis 2).

Interestingly, the correlation between item peak locations at pre and post tests within the unconstrained heterogeneous model (model 1) was 0.983, strongly supporting a common and stable structure of emotion locations along a valence dimension. Figure 14 illustrates both this unique location feature and the variation of response intensity from one time of measurement to the other, by superimposing both response subsets and the corresponding response curves. Both response curves on each plot peak at the same location along the latent dimension ( $x$-axis), but not at the same amplitude level (y-axis). The common ordering of (noninverted) items was: Depressed (-2.525), Sad (-2.451), Upset (-2.094), Tense (-1.638), Stressed (-1.498), Nervous (1.424), Stimulated (0.934), Alert (1.885), Cheerful (2.259), Happy (2.562), Pleased (2.579), Serene (2.734), supporting the hypothesis of a passive-negative to passive-positive continuum, more active states lying in between. Note that this interpretation does not rely on inverted items (Relaxed, Calm, Bored, Tired) location parameters, as for them these $\delta_{j}$ parameters correspond to a minimum (and not a maximum) of response intensity, as expected. A striking property of these four items, from the HUME perspective, is that they may receive (moderately to strong) positive agreement on both ends of the valence dimension.

That the homogeneous model is rejected suggests that, while emotion ideal locations are stable, emotional response amplitudes may vary from one time to the other, and more generally following context. On the whole, some emotions were more highly rated after the exam (Depressed, Sad, Upset) than before, and conversely for other emotions (Tense, Stressed, Stimulated, Alert, Happy, Pleased), suggesting that students tended to be more depressed, less stimulated and less happy after the exam.

Note that some caution must be taken in examining these plots. As a result of the asymmetry of the beta distribution, the expected response (lying on the response curve) will always lie at some distance of the mode (highest density response area), for any $\theta$ mood state along the $x$-axis. The expected rating curve as a whole is thus not expected to pass "through the middle of the data" (but a modal response curve would, see Noel, 2014 for details), as is familiar with a gaussian model. This is made visible on the plots by adding a LOWESS smoothing of observed responses against estimated mood states (dotted lines). Because this non-parametric regression minimizes squared distances to the estimated curves (very much like a gaussian regression), it is much more affected by density and may be viewed as a modal regression. This is helpful to see that, with no parametric constraint, the $U$ and inverted-U shaped patterns do appear.

\section{Discussion}

Kuppens et al. (2013) called for an idiographicnomothetic integration in the modeling of emotion. We believe that the HUME model brings this necessary integration. The particular form of the final model, with fixed emotion locations (the nomothetic part), but allowing for change in emotional response intensity following time and possibly contexts (the idiographic part), operationalizes a natural distinction between mood and emotion: Mood may be defined as the location of a person on the latent continuum (on the $x$-axis), while emotion is characterized by a global response level (on the $y$-axis), possibly modulated by personality or contextual elements. Affect may then be viewed as a generic concept for the whole dynamical system embedding mood values, intensity of emotional responses, and their joint variations.

This model states that human emotions may, at a functional level, be represented on a single dimension. Along this dimension, responses are assumed to vary following U- and inverted $\mathrm{U}$ patterns. Given the availability of unfolding software for both categorical (Chalmers, 2012; Roberts, Fang, Cui, \& Wang, 2006) and continuous data (Noel, 2014), this hypothesis may be easily tested on any emotion dataset, provided low arousal items are properly inverted. This does not mean that positive emotionality, negative emotionality and arousal are not biologically discriminable concepts, but that their dynamic interplay has a one-dimensional state space. So the HUME is clearly a dimensional model, but not a factor model. Some arguments have been provided in this pa- 

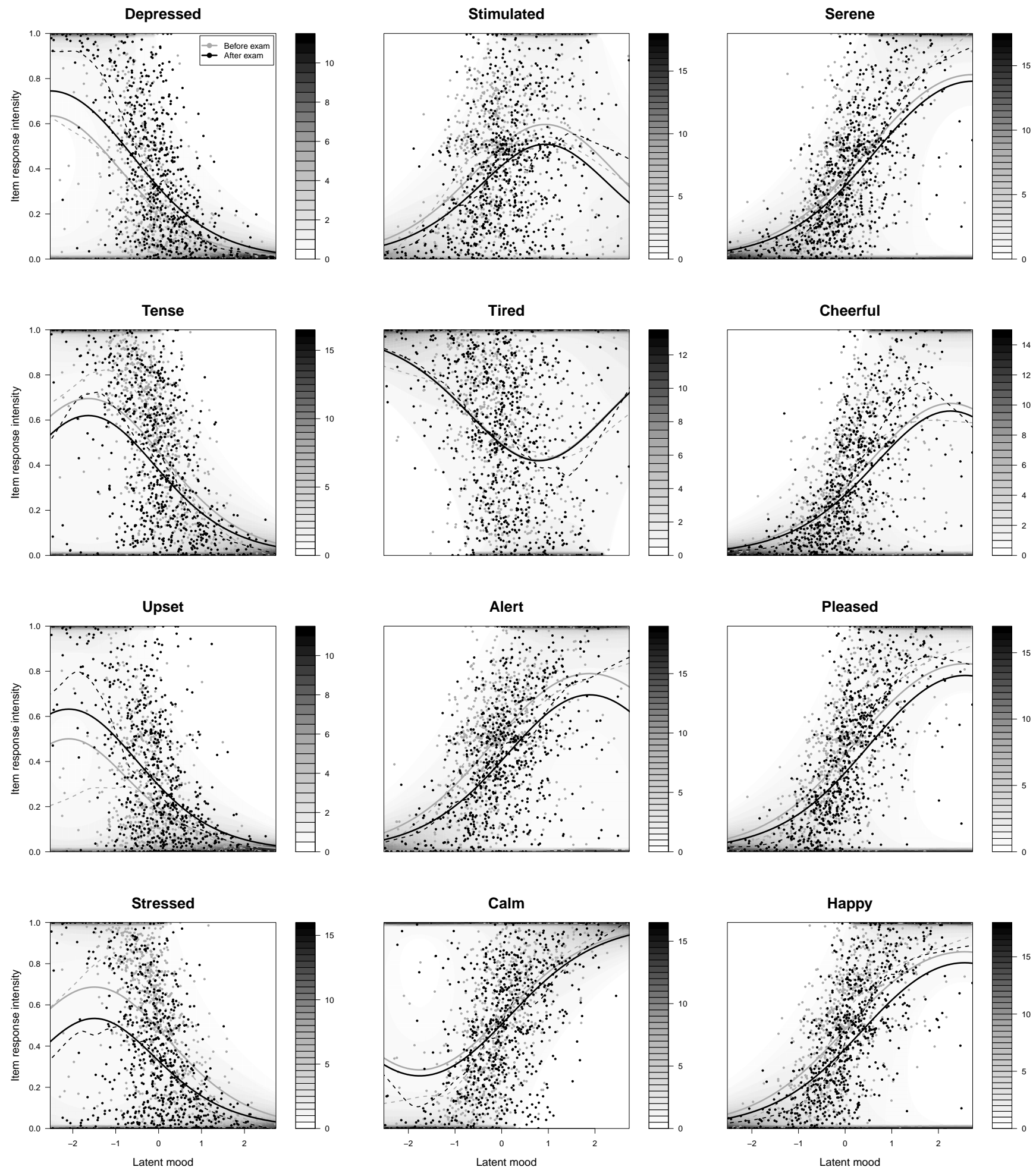

Figure 14. Pre and post exam HUME estimated response functions (grey and black plain lines) for 12 sample items. A level plot of response densities, as predicted from the model, has been added in the background, along with a non-parametric regression (LOWESS smoothing) of observed responses on the estimated latent scores (dotted lines). 
per that factors emerging from PCA studies are, at least partially, artefactual by nature. In this line, the unique dimension recovered in the analysis above is not so much a valence dimension as a latent control variable, as both valence and arousal depend upon it. This is in agreement with biological findings that elementary components of emotion functionally interact in a complex manner and cannot be simply located in the brain (Lindquist, Wager, Kober, Bliss-Moreau, \& Barrett, 2012).

Because it is unidimensional, the HUME model does not bring support for any categorical model or typology of basic emotions. But it may be viewed as a possible reconciliation of both category and dimensional approaches, by considering emotion categories as successive qualitative intervals along the latent dimension. For instance, using the HUME estimated response functions for an arbitrary subset of emotions, those points where the functions intersect could be used as intercategory thresholds. An illustration is provided on Figure 15 where three thresholds, based on the Depression, Stressed, Stimulated and Serene items characteristic curves, and their intersection, could be used to define a four-category system: Negative-Passive, Negative-Active, Positive-Active and Positive-Passive emotions. In the same vein, this would lead to expect that the six commonly distinguished basic emotions (Ekman \& Friesen, 1971) unfold in order along the HUME dimension as: Sadness, disgust, angriness/fear, surprise, and joy, as examination of Mikels and colleagues (Mikels et al., 2005) emotion categorization of the IAPS pictures suggests (see Figure 13, right panel). Any such categorization attempt is of course largely arbitrary, and is indicated here for convenience, as the true latent structure is believed to be continuous by nature.

The hypothesis of unidimensionality of the emotional dynamic space has consequences on the way emotions should be measured. There is a need for a good measure of mood and emotional states as soon as their impact on any behavior is to be assessed, and this has been done in an impressive variety of applied contexts. Such an influence has been reported on examination performance (A. M. Lane, Whyte, Terry, \& Nevill, 2005), consumer's product preference (White \& McFarland, 2009), perceived risk and choice (Fedorikhin \& Cole, 2004), risky driving (Hu, Xie, \& Li, 2013), sport performance (A. Lane \& Terry, 2000), truth judgments (Koch \& Forgas, 2012), impression formation and its persistence (Forgas, 2011), or evaluation of ingroup and outgroup members (Ziegler \& Burger, 2011), to name but a few. Measurement of affect is also of importance in clinical contexts, for understanding the clinical distinction between anxiety and depression or even diagnose depression (Watson \& NaragonGainey, 2010). Use of separate measures for positive and negative emotionality (Watson, Clark, \& Tellegen, 1988) is common practice in these studies, but our findings suggest that a more integrated, one-dimensional measure is possible.

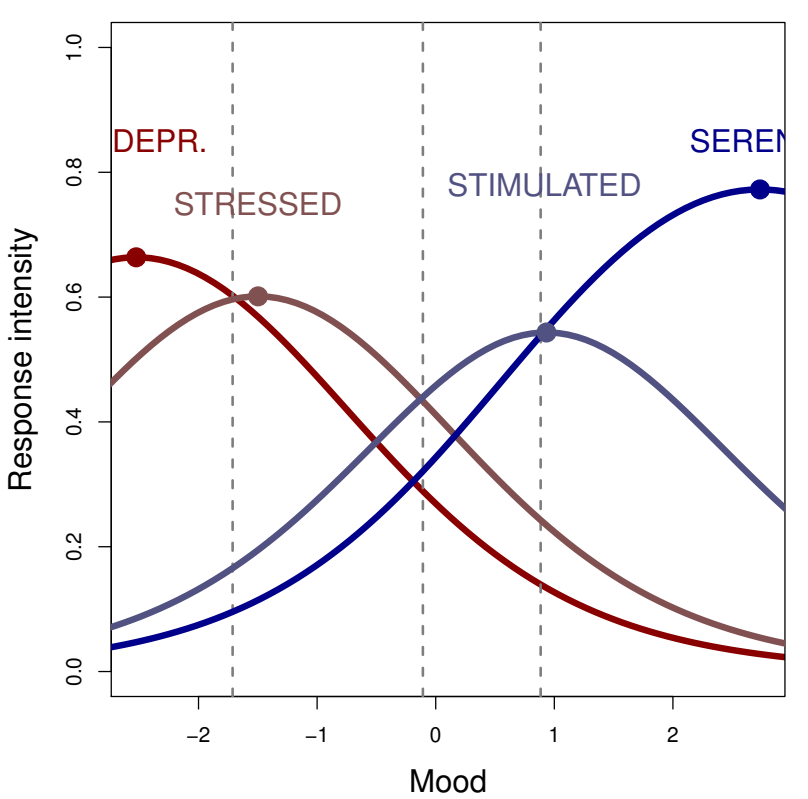

Figure 15. A reconciliation of the dimensional and the categorical views of emotion. Dotted lines mark those points where responses to two consecutive items are equal, which may help define emotion categorization.

For practical purposes, we found that a simple weighted average of the item peak locations given above, using subjects' normalized ratings as weights, provides scores that highly correlate with the unfolding scores $(r=0.90, N=$ 746, $p<0.0001$ ), as recovered in Study 4. A practical example is detailed in Table 1: A set of raw ratings $x_{j}$, from one of the subject in Study 4 (column 3), initially ranging from 0 to 480 , is first transformed into normalized ratings (i.e. divided by their sum, 2929). These are used as weights in a barycentric estimation of this subject's location along the latent continuum, by multiplication with item locations. The basic idea is that the subject should be located close to those items to which he/she gave high ratings. The approximate HUME score (noted as $h$-score) is $h=1.0742$ (close to the estimated value 0.916 found using the full model specification). Hopefully such a simple scoring procedure could be useful in future emotion research.

Finally, the HUME hypothesis may also shed a new light on why depressive states may be so difficult to cure. If the single-peaked variation of arousal, as a function of valence, is correct, then moving from negative to positive extreme states of valence implies a local increase in arousal. And this is likely to be difficult in case of severe loss of energy, as is common in depression states. This particular combination of low-energy and negative mood may explain why depression may be an (almost) stable state, or attractor, in the valence- 


\begin{tabular}{ccccc}
\hline Item & Location & Raw ratings & Normalized rating & Weighted locations \\
$j$ & $\delta_{j}$ & $x_{j}$ & $w_{j}=x_{j} / \sum_{k} x_{k}$ & $w_{j} \delta_{j}$ \\
\hline Depressed & -2.525 & 112 & 0.0382 & -0.0965 \\
Sad & -2.451 & 112 & 0.0382 & -0.0936 \\
Upset & -2.094 & 101 & 0.0345 & -0.0722 \\
Tense & -1.638 & 190 & 0.0649 & -0.1063 \\
Stressed & -1.498 & 93 & 0.0318 & -0.0476 \\
Nervous & -1.424 & 195 & 0.0666 & -0.0949 \\
Stimulated & 0.934 & 325 & 0.1110 & 0.1037 \\
Alert & 1.885 & 294 & 0.1004 & 0.1892 \\
Cheerful & 2.259 & 404 & 0.1379 & 0.3116 \\
Happy & 2.562 & 430 & 0.1468 & 0.3761 \\
Pleased & 2.579 & 440 & 0.1502 & 0.3873 \\
Serene & 2.734 & 233 & 0.0795 & 0.2174 \\
\hline & Sum: & 2929 & 1.0000 & $h=1.0742$
\end{tabular}

Table 1

Computing an approximate HUME score (or h-score) from observed responses (inverted items excluded), as a weighted average of item locations.

activation dynamical system. Conversely, the HUME also predicts that, from a moderately negative state, increasing arousal (for instance by physical exercise, if possible) should be associated with a movement toward more positive mood states, which has been documented in the sport and exercise literature (Byrne \& Byrne, 1993). But this should not be observed among people already in a good mood, a differential effect that has also been reported (A. Lane \& Lovejoy, 2001). More work is needed to see if any kind of causal influence of one component (valence or activation) on the other really exists, and if so, if it is fully bidirectional, or if one of them may have a stronger impact on the other.

\section{References}

Acton, G. S., \& Revelle, W. (2004). Evaluation of Ten Psychometric Criteria for Circumplex Structure. Methods of Psychologcal Research, 9, 1-27.

Andrich, D., \& Luo, G. (1993). A hyperbolic cosine latent trait model for unfolding dichotomous single-stimulus responses. Applied Psychological Measurement, 17, 253-276.

Barrett, L. F., \& Russell, J. A. (1998). Independence and bipolarity in the structure of current affect. Journal of Personality and Social Psychology, 74(4), 967-984.

Barrett, L. F., \& Russell, J. A. (1999). The structure of current affect: Controversies and emerging consensus. Current Directions in Psychological Science, 8(1), 1014.

Beedie, C. J., Terry, P. C., Lane, A. M., \& Devonport, T. J. (2011). Differential assessment of emotions and moods: Development and validation of the emotion and mood components of anxiety questionnaire. Personality and Individual Differences, 50(2), 228-233.
Benzécri, J. (1973-1982). L’analyse des données. Paris: Dunod.

Bradley, M. M., \& Lang, P. J. (1994). Measuring emotion: The self-assessment manikin and the semantic differential. Journal of Behavior Therapy and Experimental Psychiatry, 25(1), 49-59.

Bradley, M. M., \& Lang, P. J. (2007). The International Affective Picture System (IAPS) in the study of emotion and attention. In J. A. Coan \& J. J. B. Allen (Eds.), Handbook of emotion elicitation and assessment (pp. 29-46). Oxford University Press.

Briesemeister, B. B., Kuchinke, L., \& Jacobs, A. M. (2012). Emotional valence: A bipolar continuum or two independent dimensions? SAGE Open, 2(4).

Byrne, A., \& Byrne, D. (1993). The effect of exercise on depression, anxiety and other mood states: a review. Journal of Psychosomatic Research, 37(6), 565-574.

Cacioppo, J. T., \& Berntson, G. G. (1994). Relationship between attitudes and evaluative space: A critical review, with emphasis on the separability of positive and negative substrates. Psychological Bulletin, 115(3), 401.

Cattell, R. (1957). Personality and motivation structure and measurement. New York: World Book.

Chalmers, R. (2012). mirt: A multidimensional item response theory package for the R environment. Journal of Statistical Software, 48(6), 1-29. Retrieved from http://www. jstatsoft.org/v48/i06/

Citron, F. M., Gray, M. A., Critchley, H. D., Weekes, B. S., \& Ferstl, E. C. (2014). Emotional valence and arousal affect reading in an interactive way: Neuroimaging evidence for an approach-withdrawal framework. Neuropsychologia, 79-89.

Citron, F. M., Weekes, B. S., \& Ferstl, E. C. (2014). Arousal and emotional valence interact in written word recog- 
nition. Language, Cognition and Neuroscience(aheadof-print), 1-11.

Coombs, C. (1950). Psychological scaling without a unit of measurement. Psychological Review, 57(3), 145-158.

Davison, M. L. (1977). On a metric, unidimensional unfolding model for attitudinal and developmental data. Psychometrika, 42(4), 523-548.

Descartes, R. (1649). Les passions de l'âme. Paris: Henri Le Gras.

Dony, R. (2000). Karhunen-loève transform. In K. R. Rao \& P. Yip (Eds.), The transform and data compression handbook. Boca Raton, FL, USA: CRC Press, Inc.

Ekkekakis, P., Hall, E. E., \& Petruzzello, S. J. (2005). Evaluation of the circumplex structure of the activation deactivation adjective check list before and after a short walk. Psychology of Sport and Exercise, 6(1), 83-101.

Ekkekakis, P., Hall, E. E., Petruzzello, S. J., et al. (1999). Measuring state anxiety in the context of acute exercise using the state anxiety inventory: An attempt to resolve the brouhaha. Journal of Sport and Exercise Psychology, 21, 205-229.

Ekman, P., \& Friesen, W. (1971). Constants across cultures in the face and emotion. Journal of Personality and Social Psychology, 17(2), 124-129.

Embretson, S., \& Reise, S. (2000). Item response theory for psychologists. Mahwah, NJ: L. Erlbaum Associates.

Everitt, B. S. (2005). Horseshoe pattern. In B. Everitt \& D. Howell (Eds.), Encyclopedia of statistics in behavioral science (p. 889). Chichester: John Wiley \& Sons, Ltd.

Fedorikhin, A., \& Cole, C. (2004). Mood effects on attitudes, perceived risk and choice: Moderators and mediators. Journal of Consumer Psychology, 14(1-2), 2-12.

Feldman Barrett, L., \& Russell, J. (1998). Independence and bipolarity in the structure of current affect. Journal of Personality and Social Psychology, 74(4), 967.

Ferrando, P. J. (2001). A nonlinear congeneric model for continuous item responses. British Journal of Mathematical and Statistical Psychology, 54(2), 293-313.

Forgas, J. (2011). Can negative affect eliminate the power of first impressions? affective influences on primacy and recency effects in impression formation. Journal of Experimental Social Psychology, 47(2), 425-429.

Green, D. P., Goldman, S. L., \& Salovey, P. (1993). Measurement error masks bipolarity in affect ratings. Journal of Personality and Social Psychology, 64(6), 10291041.

Greenacre, M. (2007). Correspondence analysis in practice, second edition. Taylor \& Francis.

Guttman, L. (1954). A new approach to factor analysis: The radex. In P. F. Lazarsfeld (Ed.), Mathematical thinking in the social sciences (pp. 258-348). Glencoe, IL: Free Press.
Hastie, T., \& Stuetzle, W. (1989). Principal curves. Journal of the American Statistical Association, 84(406), 502516.

Hastie, T., \& Weingessel, A. (2013). princurve: Fits a principal curve in arbitrary dimension [Computer software manual]. Retrieved from http : //CRAN . R-project . org/package=princurve (R package version 1.1-12)

Horn, J. L. (1965). A rationale and test for the number of factors in factor analysis. Psychometrika, 30(2), 179185.

Hu, T.-Y., Xie, X., \& Li, J. (2013). Negative or positive? the effect of emotion and mood on risky driving. Transportation Research Part F: Traffic Psychology and Behaviour, 16, 29-40.

Izard, C. E. (1971). The face of emotion. New York: Appleton-Century-Crofts.

Jerome, G. J., Marquez, D. X., McAuley, E., Canaklisova, S., Snook, E., \& Vickers, M. (2002). Self-efficacy effects on feeling states in women. International Journal of Behavioral Medicine, 9(2), 139-154.

Kang, C., Wang, Z., Surina, A., \& Lu, W. (2014). Immediate emotion-enhanced memory dependent on arousal and valence: The role of automatic and controlled processing. Acta Psychologica, 150(0), 153-160.

Koch, A., \& Forgas, J. (2012). Feeling good and feeling truth: The interactive effects of mood and processing fluency on truth judgments. Journal of Experimental Social Psychology, 48(2), 481-485.

Kuppens, P., Tuerlinckx, F., Russell, J. A., \& Barrett, L. F. (2013). The relation between valence and arousal in subjective experience. Psychological Bulletin, 139(4), 917-940.

Lane, A., \& Lovejoy, D. (2001). The effects of exercise on mood changes: The moderating effect of depressed mood. Journal of Sports Medicine and Physical Fitness, 41(4), 539-545.

Lane, A., \& Terry, P. (2000). The nature of mood: Development of a conceptual model with a focus on depression. Journal of Applied Sport Psychology, 12(1), 16-33.

Lane, A. M., Whyte, G. P., Terry, P. C., \& Nevill, A. M. (2005). Mood, self-set goals and examination performance: the moderating effect of depressed mood. Personality and Individual Differences, 39(1), 143-153.

Lang, P. J., Bradley, M. M., \& Cuthbert, B. N. (2008). International Affective Picture System (IAPS): Affective ratings of pictures and instruction manual (Tech. Rep. No. A-8). Gainesville, FL.: University of Florida.

Larsen, J. T., \& McGraw, A. P. (2011). Further evidence for mixed emotions. Journal of Personality and Social Psychology, 100(6), 1095-1110.

Larsen, J. T., McGraw, A. P., \& Cacioppo, J. T. (2001). Can 
people feel happy and sad at the same time? Journal of Personality and Social Psychology, 81(4), 684-696.

Lazarus, R., \& Folkman, S. (1984). Stress, appraisal, and coping. New York: Springer.

Lindquist, K. A., Wager, T. D., Kober, H., Bliss-Moreau, E., \& Barrett, L. F. (2012). The brain basis of emotion: A meta-analytic review. Behavioral and Brain Sciences, 35(03), 121-143.

Maraun, M. D., \& Rossi, N. T. (2001). The extra-factor phenomenon revisited: Unidimensional unfolding as quadratic factor analysis. Applied Psychological Measurement, 25(1), 77-87.

Mellenbergh, G. J. (1994). A unidimensional latent trait model for continuous item responses. Multivariate Behavioral Research, 29(3), 223-236.

Mikels, J. A., Fredrickson, B. L., Larkin, G. R., Lindberg, C. M., Maglio, S. J., \& Reuter-Lorenz, P. A. (2005). Emotional category data on images from the International Affective Picture System. Behavior Research Methods, 37(4), 626-630.

Noel, Y. (1999). Recovering latent unimodal patterns of change by unfolding analysis: Application to smoking cessation. Psychological Methods, 4, 173-191.

Noel, Y. (2014). A beta unfolding model for continuous bounded responses. Psychometrika, 79(4).

Noel, Y., \& Dauvier, B. (2007). A beta item response model for continuous bounded responses. Applied Psychological Measurement, 31(1), 47-73.

Plutchik, R. (2002). Emotions and life: Perspectives from psychology, biology, and evolution. Washington D.C.: American Psychological Association.

Plutchik, R., \& Conte, H. (1997). Circumplex models of personality and emotions. American Psychological Association.

Posner, J., Russell, J. A., Gerber, A., Gorman, D., Colibazzi, T., Yu, S., ... Peterson, B. S. (2009). The neurophysiological bases of emotion: An fmri study of the affective circumplex using emotion-denoting words. $\mathrm{Hu}$ man Brain Mapping, 30(3), 883-895.

Rafaeli, E., \& Revelle, W. (2006). A premature consensus: Are happiness and sadness truly opposite affects? $\mathrm{Mo}$ tivation and Emotion, 30(1), 1-12.

Rasch, G. (1960). Probabalistic models for some intelligence and attainment tests. Danmarks paedagogiske institut.

Roberts, J. S., Donoghue, J. R., \& Laughlin, J. E. (2000). A general item response theory model for unfolding unidimensional polytomous responses. Applied Psychological Measurement, 24, 3-32.

Roberts, J. S., Fang, H., Cui, W., \& Wang, Y. (2006). Ggum2004: A windows-based program to estimate parameters in the generalized graded unfolding model. Applied Psychological Measurement, 30, 64-65.

Robinson, M. D., Storbeck, J., Meier, B. P., \& Kirkeby, B. S.
(2004). Watch out! that could be dangerous: Valencearousal interactions in evaluative processing. Personality and Social Psychology Bulletin, 30(11), 14721484.

Russell, J. (1979). Affective space is bipolar. Journal of Personality and Social Psychology, 37(3), 345-356.

Russell, J. (1980). A circumplex model of affect. Journal of Personality and Social Psychology, 39(6), 11611178.

Russell, J., \& Carroll, J. (1999). On the bipolarity of positive and negative affect. Psychological bulletin, 125(1), 3.

Russell, J., Lewicka, M., \& Niit, T. (1989). A cross-cultural study of a circumplex model of affect. Journal of Personality and Social Psychology, 57(5), 848-856.

Seligman, M. E. (1972). Learned helplessness. Annual Review of Medicine, 23(1), 407-412.

Sheth, B. R., \& Pham, T. (2008). How emotional arousal and valence influence access to awareness. Vision Research, 48(23), 2415-2424.

Smithson, M., \& Verkuilen, J. (2006). A better lemonsqueezer? Maximum likelihood regression with betadistributed dependent variables. Psychological Methods, 11(1), 54-71.

Tellegen, A., Watson, D., \& Clark, L. A. (1999). On the dimensional and hierarchical structure of affect. Psychological Science, 10(4), 297-303.

Tibshirani, R. (1992). Principal curves revisited. Statistics and Computing, 2, 183-190.

Tomkins, S. (1962). Affect, imagery, consciousness: Vol. I. the positive affects. New York: Springer.

van der Linden, W. J., \& Hambleton, R. (1997). Handbook of modern item response theory. New York: Springer.

Van Schuur, W., \& Kruijtbosch, M. (1995). Measuring subjective well-being: Unfolding the Bradburn Affect Balance Scale. Social Indicators Research, 36(1), 4974.

van Schuur, W. H., \& Kiers, H. A. L. (1994). Why factor analysis often is the incorrect model for analyzing bipolar concepts, and what model to use instead. Applied Psychological Measurement, 18(2), 97-110.

Verkuilen, J., \& Smithson, M. (2012). Mixed and mixture regression models for continuous bounded responses using the beta distribution. Journal of Educational and Behavioral Statistics, 37(1), 82-113.

Watson, D. (2000). Mood and temperament. New York: Guilford Press.

Watson, D., \& Clark, L. A. (1997). Measurement and mismeasurement of mood: Recurrent and emergent issues. Journal of Personality Assessment, 68(2), 267296.

Watson, D., Clark, L. A., \& Tellegen, A. (1988). Development and validation of brief measures of positive and negative affect: The PANAS scales. Journal of Per- 
sonality and Social Psychology, 54, 1063-1070.

Watson, D., \& Naragon-Gainey, K. (2010). On the specificity of positive emotional dysfunction in psychopathology: Evidence from the mood and anxiety disorders and schizophrenia/schizotypy. Clinical Psychology Review, 30(7), 839-848.

Watson, D., \& Tellegen, A. (1985). Toward a consensual structure of mood. Psychological bulletin, 98(2), 219235.

Watson, D., \& Tellegen, A. (1999). Issues in dimensional structure of affect - effects of descriptors, measurement error, and response formats: Comment on Russell and Carroll (1999). Psychological Bulletin, 125(5), 601-610.

White, K., \& McFarland, C. (2009). When are moods most likely to influence consumers' product preferences? the role of mood focus and perceived relevance of moods. Journal of Consumer Psychology, 19(3), 526-536.

Wright, B., \& Masters, G. (1982). Rating scale analysis. Chicago: MESA Press.

Yik, M. S. M., Russell, J. A., \& Barrett, L. F. (1999). Structure of self-reported current affect: Integration and beyond. Journal of Personality and Social Psychology, 77(3), 600-619.

Ziegler, R., \& Burger, A. (2011). Mood and the impact of individuating information on the evaluation of ingroup and outgroup members: The role of mood-based expectancies. Journal of Experimental Social Psychology, 47(5), 1000-1006.

Zigmond, A., \& Snaith, R. (1983). The hospital anxiety and depression scale. Acta Psychiatrica Scandinavica, 67, 361-370. 